\title{
DIRICHLET PROBLEM AT INFINITY FOR HARMONIC MAPS: RANK ONE SYMMETRIC SPACES
}

\author{
HAROLD DONNELLY
}

\begin{abstract}
Given a symmetric space $M$, of rank one and noncompact type, one compactifies $M$ by adding a sphere at infinity, to obtain a manifold $M^{\prime}$ with boundary. If $\bar{M}$ is another rank one symmetric space, suppose that $f: \partial M^{\prime} \rightarrow \partial \bar{M}^{\prime}$ is a continuous map. The Dirichlet problem at infinity is to construct a proper harmonic map $u: M \rightarrow \bar{M}$ with boundary values $f$. This paper concerns existence, uniqueness, and boundary regularity for this Dirichlet problem.
\end{abstract}

\section{INTRODUCTION}

Let $M$ and $\bar{M}$ be complete simply connected manifolds of strictly negative curvature. One may compactify $M$ and $\bar{M}$, using asymptotic classes of geodesic rays, by adding spheres at infinity. We denote the compactifications by $M^{\prime}$ and $\bar{M}^{\prime}$, and the spheres added at infinity by $\partial M^{\prime}, \partial \bar{M}^{\prime}$. Suppose that $f: \partial M^{\prime} \rightarrow \partial \bar{M}^{\prime}$ is a continuous map. The Dirichlet problem at infinity consists of finding a harmonic map $u: M \rightarrow \bar{M}$, with boundary values $f$ at infinity. Here one means that $u \in C^{2}(M, \bar{M}) \cap C^{0}\left(M^{\prime}, \bar{M}^{\prime}\right)$, and the boundary values $f$ are taken continuously. In general, the Dirichlet problem at infinity seems to be quite difficult. If $M$ and $\bar{M}$ both have constant negative curvature, then $\mathrm{Li}$ and Tam $[8,9]$ have proved a number of significant results, concerning uniqueness, existence, and boundary regularity. Our plan is to extend this discussion to the context of rank one symmetric spaces.

Suppose now that $M$ and $\bar{M}$ are rank one symmetric spaces of noncompact type. In the unbounded model, $M$ is realized as $R^{+} \times N$, where $R^{+}$is the positive real line and $N$ is a two term nilpotent group. The Lie algebra of $N$ decomposes as $\mathfrak{n}=\mathfrak{n}_{1} \oplus \mathfrak{n}_{2}$, where $\mathfrak{n}_{2}$ is central in $\mathfrak{n}$ and $\left[\mathfrak{n}_{1}, \mathfrak{n}_{1}\right] \subset \mathfrak{n}_{2}$. For the exceptional case, of constant negative curvature on $M$, we adopt the convention that $\mathfrak{n}_{1}$ is the entire abelian Lie algebra and $\mathfrak{n}_{2}$ is empty. In the unbounded model $R^{+} \times N$, the metric of $M$ is realized as a doubly warped product [1]:

$$
g_{M}=\left(\frac{d y}{y}\right)^{2}+y^{-2} g_{\mathfrak{n}_{1}}+y^{-4} g_{\mathfrak{n}_{2}} .
$$

Received by the editors July 19, 1993.

1991 Mathematics Subject Classification. Primary 58E20.

Partially supported by NSF Grant DMS-9200225. 
Here $y \in R^{+}$is the coordinate on the first factor of $R^{+} \times N$. Via Cayley transform, this provides local coordinate charts at the boundary $\partial M^{\prime}$ of the compactification. Of course, the same discussion applies to $\bar{M}^{\prime}$. To formulate our results, we introduce indices $0 \leq j \leq n_{1}+n_{2}$. The index 0 refers to $\partial / \partial y$, the indices $1 \leq j \leq n_{1}$ allude to the $g_{\mathfrak{n}_{1}}$ part of (1.1); and the indices $n_{1}+1 \leq j \leq n_{1}+n_{2}$ refer to the $g_{n_{2}}$ part of (1.1). On $\bar{M}$, we use corresponding Greek indices $0 \leq \alpha \leq \bar{n}_{1}+\bar{n}_{2}$.

Our first observation is that if $f$ is the boundary value of a harmonic map $u \in C^{2}(M, \bar{M}) \cap C^{1}\left(M^{\prime}, \bar{M}^{\prime}\right)$, then $f_{j}^{\gamma}=0,1 \leq j \leq n_{1}, \bar{n}_{1}+1 \leq \gamma \leq \bar{n}_{1}+\bar{n}_{2}$. Here $f_{j}^{y}$ are the components of the differential of $f$. By contrast, Li and Tam [8] proved that, for spaces of constant negative curvature, any $f$ with nonvanishing energy density can occur as the boundary value of a $C^{1}\left(M^{\prime}, \bar{M}^{\prime}\right)$ harmonic map. If $h$ is a harmonic self-map of the unit ball in $C^{n}$, with its Bergman metric, the condition $f_{j}^{\gamma}=0$ means that $f$ is a contact transformation.

We now describe our uniqueness results. Suppose that both $h$ and $\hat{h}$ are proper harmonic maps between rank one symmetric space $M$ and $\bar{M}$. Assume $h, \hat{h} \in C^{2}\left(M^{\prime}, \bar{M}^{\prime}\right)$ have the same boundary value $f: \partial M^{\prime} \rightarrow \partial \bar{M}^{\prime}$, which satisfies

$$
\sum_{j=n_{1}+1}^{n_{1}+n_{2}} \sum_{\gamma=\bar{n}_{1}+1}^{\bar{n}_{1}+\bar{n}_{2}} f_{j}^{\gamma} f_{j}^{\gamma}>0 .
$$

Then $h$ and $\hat{h}$ are identical. If the range $\bar{M}$ has constant negative curvature, then one only needs $h, \hat{h} \in C^{1}\left(M^{\prime}, \bar{M}^{\prime}\right)$. If the common boundary value satisfies

$$
\sum_{j=1}^{n_{1}} \sum_{\gamma=1}^{\bar{n}_{1}} f_{j}^{\gamma} f_{j}^{\gamma}>0
$$

then $h=\hat{h}$. This last result was proved by Li and Tam in [8] when both $M$ and $\bar{M}$ have constant negative curvature, and their proof is similar to ours.

Our basic existence result assumes that one is given boundary data $f \in$ $C^{2, \varepsilon}\left(\partial M^{\prime}, \partial \bar{M}^{\prime}\right), 0<\varepsilon<1$, so that $f_{j}^{\gamma}=0,1 \leq j \leq n_{1}, \bar{n}_{1}+1 \leq \gamma \leq \bar{n}_{1}+$ $\bar{n}_{2}$, and $\sum_{j=n_{1}+1}^{n_{1}+n_{2}} \sum_{\gamma=\bar{n}_{1}+1}^{\bar{n}_{1} \bar{n}_{2}} f_{j}^{\gamma} f_{j}^{\gamma}>0$. We construct a harmonic map $u: M \rightarrow$ $\bar{M}$, which assumes the boundary values $f$ continuously. If the range $\bar{M}$ has constant negative curvature, it is enough to assume $f \in C^{1, \varepsilon}\left(\partial M^{\prime}, \partial \bar{M}^{\prime}\right)$, $0<\varepsilon<1$, and $\sum_{j=1}^{n_{1}} \sum_{\gamma=1}^{\bar{n}_{1}} f_{j}^{\gamma} f_{j}^{\gamma}>0$. We prove the existence of a harmonic map $u$, which assumes the boundary values $f$ continuously. If both the domain and range have constant negative curvature, this again reduces to a result of [8], where similar arguments were employed.

For our regularity results, we assume that $f \in C^{l+2, \varepsilon}\left(\partial M^{\prime}, \partial \bar{M}^{\prime}\right), 0 \leq l<$ $n_{1}+2 n_{2}, 0<\varepsilon<1$, satisfies $f_{j}^{\gamma}=0,1 \leq j \leq n_{1}, \bar{n}_{1}+1 \leq \gamma \leq \bar{n}_{1}+\bar{n}_{2}$, and $\sum_{j=n_{1}+1}^{n_{1}+n_{2}} \sum_{\gamma=\bar{n}_{1}+1}^{\bar{n}_{1}+\bar{n}_{2}} f_{j}^{\gamma} f_{j}^{\gamma}>0$. We prove that there is a harmonic map, $u: M \rightarrow$ $\bar{M}^{\prime}, u \in C^{k+1, \bar{\varepsilon}}\left(M^{\prime}, \bar{M}^{\prime}\right) \cap C^{2}(M, \bar{M})$, with boundary values $f$, when $\bar{\varepsilon}<\varepsilon$ and $-2 \leq 2 k<l-1$. Although the factor 2 in $2 k$ is not appealing, it may be needed. In [4], Graham studied the model linear problem of the Bergman Laplacian on the unit ball in $C^{n}$, where similar difficulties appear. If both $M$ 
and $\bar{M}$ have constant negative curvature, then one instead assumes that $f \in$ $C^{l+1, \varepsilon}\left(\partial M^{\prime}, \partial \bar{M}^{\prime}\right), 0<\varepsilon<1,0 \leq l<n_{1}$, and $\sum_{j=1}^{n_{1}} \sum_{\gamma=1}^{\bar{n}_{1}} f_{j}^{\gamma} f_{j}^{\gamma}>0$. Then there exists a harmonic map $u: M \rightarrow \bar{M}, u \in C^{l+1, \varepsilon}\left(M^{\prime}, \bar{M}^{\prime}\right) \cap C^{2}(M, \bar{M})$, with boundary values $f$. This was proved earlier in [8]. However, our method of proof is somewhat different. We replace the use of conformality to the Euclidean Laplacian with ideas based upon the facts that our spaces $M, \bar{M}$ admit transitive isometry groups.

\section{TENSION IN AN ADAPTED FRAME FIELD}

Let $h: M \rightarrow \bar{M}$ be a $C^{2}$ map between Riemannian manifolds $M$ and $\bar{M}$. The differential $d h$ may be regarded as a section of $T^{*} M \otimes h^{-1} T \bar{M}$. The bundle $T^{*} M \otimes h^{-1} T \bar{M}$ admits a connection $\nabla$ induced by the Levi-Civita connection on $M$ and the pull-back of the Levi-Civita connection on $\bar{M}$. One defines the tension $\tau(h)=\operatorname{Tr}(\nabla d h)$. For later reference, we compute $\tau(h)$ explicitly, especially in the case where $M$ and $\bar{M}$ are rank one symmetric spaces of noncompact type.

Suppose that $e_{i}$ is a local frame field on $T M$, with dual coframe field $e_{i}^{*}, i=1,2, \ldots, \operatorname{dim} M$. Let $f_{\alpha}$ be a local frame field for $T \bar{M}, \alpha=$ $1,2, \ldots, \operatorname{dim} \bar{M}$. We do not assume that either $e_{i}$ or $f_{\alpha}$ is orthonormal. We may denote the differential of $h$ as $d h=h_{i}^{\alpha} e_{i}^{*} \otimes f_{\alpha}$, where one sums over both the indices $i$ and $\alpha$. Let $g^{i j}=\left\langle e_{i}^{*}, e_{j}^{*}\right\rangle$ and $h_{i j}^{\alpha}=e_{j} h_{i}^{\alpha}$. Calculating from the definition yields

$$
\begin{aligned}
\tau(h) & =\operatorname{Tr}(\nabla d h)=\operatorname{Tr}\left(e_{j}^{*} \otimes \nabla_{e_{j}}\left(h_{i}^{\alpha} e_{i}^{*} \otimes f_{\alpha}\right)\right) \\
& =g^{i j} h_{i j}^{\alpha} f_{\alpha}+h_{i}^{\alpha}\left\langle e_{j}^{*}, \nabla_{e_{j}} e_{i}^{*}\right\rangle f_{\alpha}+h_{i}^{\alpha} g^{i j} \nabla_{e_{j}} f_{\alpha}
\end{aligned}
$$

where summation is understood over $i, j$, and $\alpha$.

We rewrite the formula for $\tau(h)$ by noting that

$$
\begin{aligned}
& \left\langle e_{j}^{*}, \nabla_{e_{j}} e_{i}^{*}\right\rangle=g^{j k} e_{k}\left(\nabla_{e_{j}} e_{i}^{*}\right)=-g^{j k} e_{i}^{*}\left(\nabla_{e_{j}} e_{k}\right), \\
& \nabla_{e_{j}} f_{\alpha}=\nabla_{h_{j}^{\beta} f_{\beta}} f_{\alpha}=h_{j}^{\beta} \nabla_{f_{\beta}} f_{\alpha}=h_{j}^{\beta} f_{\gamma}^{*}\left(\nabla_{f_{\beta}} f_{\alpha}\right) f_{\gamma} .
\end{aligned}
$$

Thus $\tau(h)=\tau^{\alpha}(h) f_{\alpha}$, where

$$
\tau^{\alpha}(h)=g^{i j} h_{i j}^{\alpha}-g^{j k} e_{i}^{*}\left(\nabla_{e_{j}} e_{k}\right) h_{i}^{\alpha}+g^{i j} h_{i}^{\gamma} h_{j}^{\beta} f_{\alpha}^{*}\left(\nabla_{f_{\beta}} f_{\gamma}\right) .
$$

The summation convention is employed for the indices $i, j, k, \beta$, and $\gamma$.

The next step is to give more explicit expressions for $\tau(h)$ in the special case where $M$ on $\bar{M}$ are both rank one symmetric spaces of noncompact type. In the unbounded model, $M$ is realized as $R^{+} \times N$, where $R^{+}$is the positive real line and $N$ is a two term nilpotent Lie group. The Lie algebra of $N$ decomposes as $\mathfrak{n}=\mathfrak{n}_{1} \oplus \mathfrak{n}_{2}$, where $\mathfrak{n}_{2}$ is the central in $\mathfrak{n}$ and $\left[\mathfrak{n}_{1}, \mathfrak{n}_{1}\right] \subset$ $\mathfrak{n}_{2}$. The hyperbolic space of constant negative curvature is exceptional, and $N$ reduces to the abelian group $R^{\operatorname{dim} M-1}$. For the hyperbolic space, we adopt the convention that $\mathfrak{n}_{1}$ is the entire abelian Lie algebra and $\mathfrak{n}_{2}$ is empty. Choose an orthonormal basis $X_{1}, X_{2}, \ldots, X_{n_{1}}$ for $\mathfrak{n}_{1}$ and $Z_{1}, Z_{2}, \ldots, Z_{n_{2}}$ for $\mathfrak{n}_{2}$, relative to a left invariant metric on $N$. Here $n_{1}=\operatorname{dim} \mathfrak{n}_{1}, n_{2}=\operatorname{dim} \mathfrak{n}_{2}$, and thus $\operatorname{dim} M=n_{1}+n_{2}+1$. One has $\left[X_{i}, Z_{j}\right]=\left[Z_{j}, Z_{k}\right]=0$ and $\left[X_{i}, X_{j}\right]=$ $a_{i j}^{k} Z_{k}$, for some structure constants $a_{i j}^{k}$. A sum is understood over $k$. In the 
unbounded model $R^{+} \times N$, the metric of $M$ is realized as a doubly warped product [1]:

$$
g_{M}=\left(\frac{d y}{y}\right)^{2} \oplus y^{-2} g_{\mathfrak{n}_{1}} \oplus y^{-4} g_{\mathfrak{n}_{2}}, \quad y>0
$$

Here $y \in R^{+}$is the coordinate on the first factor of $R^{+} \times N$. Moreover, $g_{n_{1}}+g_{n_{2}}$ is a left invariant metric on $N$. Of course, the same discussion applies to $\bar{M}$, where we denote the corresponding quantities with a bar, for example $\bar{X}_{i}$ are an orthonormal basis of $\overline{\mathfrak{n}}_{1}$.

On any Riemannian manifold, with metric $g$, there is a standard elementary formula [6] for the Levi-Civita connection:

$$
\begin{aligned}
2 g\left(A, \nabla_{C} B\right)= & C g(A, B)+B g(A, C)-A g(B, C) \\
& +g(C,[A, B])+g(B,[A, C])-g(A,[B, C])
\end{aligned}
$$

where $A, B, C$ are vector fields. Using this formula, a lengthy but straightforward computation gives the connection $\nabla$, in the frame field $\partial / \partial y, X_{i}$, $Z_{j}$, of $M$ :

$$
\begin{aligned}
\nabla_{\partial / \partial y} \frac{\partial}{\partial y} & =-y^{-1} \frac{\partial}{\partial y} \\
\nabla_{X_{i}} \frac{\partial}{\partial y} & =\nabla_{\partial / \partial y} X_{i}=-y^{-1} X_{i}, \\
\nabla_{Z_{i}} \frac{\partial}{\partial y} & =\nabla_{\partial / \partial y} Z_{i}=-2 y^{-1} Z_{i}, \\
\nabla_{X_{i}} X_{j} & =y^{-1} \delta_{i j} \frac{\partial}{\partial y}+\frac{1}{2} a_{i j}^{k} Z_{k}, \\
\nabla_{Z_{i}} Z_{j} & =2 y^{-3} \delta_{i j} \frac{\partial}{\partial y} \\
\nabla_{X_{i}} Z_{j} & =\nabla_{Z_{j}} X_{i}=\frac{1}{2} y^{-2} a_{k i}^{j} X_{k} .
\end{aligned}
$$

In the exceptional case where $M$ is the hyperbolic space, there are no $Z_{i}$ 's, and (2.3) becomes

$$
\begin{aligned}
\nabla_{\partial / \partial y} \frac{\partial}{\partial y} & =-y^{-1} \frac{\partial}{\partial y} \\
\nabla_{X_{i}} \frac{\partial}{\partial y} & =\nabla_{\partial / \partial y} X_{i}=-y^{-1} X_{i} \\
\nabla_{X_{i}} X_{j} & =y^{-1} \delta_{i j} \frac{\partial}{\partial y}
\end{aligned}
$$

Of course, the frame field $\partial / \partial y, X_{i}, Z_{j}$ is orthogonal but not orthonormal for the metric $g_{M}$. Sometimes, it will be useful to employ the orthonormal 
frame field $y \partial / \partial y, y X_{i}, y^{2} Z_{j}$, where one has the corresponding expressions

$$
\begin{aligned}
& \nabla_{y \partial / \partial y}\left(y \frac{\partial}{\partial y}\right)=0, \\
& \nabla_{y X_{i}}\left(y \frac{\partial}{\partial y}\right)-\nabla_{y \partial / \partial y}\left(y X_{i}\right)=\left[y X_{i}, y \frac{\partial}{\partial y}\right]=-y X_{i}, \\
& \nabla_{y \partial / \partial y}\left(y X_{i}\right)=0, \\
& \nabla_{y^{2} Z_{i}}\left(y \frac{\partial}{\partial y}\right)-\nabla_{y \partial / \partial y}\left(y^{2} Z_{i}\right)=\left[y^{2} Z_{i}, y \frac{\partial}{\partial y}\right]=-2 y^{2} Z_{i}, \\
& \nabla_{y \partial / \partial y}\left(y^{2} Z_{i}\right)=0, \\
& \nabla_{y X_{i}}\left(y X_{j}\right)=\delta_{i j} y \frac{\partial}{\partial y}+\frac{1}{2} a_{i j}^{k} y^{2} Z_{k}, \\
& \nabla_{y^{2} Z_{i}}\left(y^{2} Z_{j}\right)=2 \delta_{i j} y \frac{\partial}{\partial y}, \\
& \nabla_{y X_{i}}\left(y^{2} Z_{j}\right)=\nabla_{y^{2} Z_{j}}\left(y X_{i}\right)=\frac{1}{2} a_{k i}^{j} y X_{k} .
\end{aligned}
$$

The advantage of the orthonormal frame field $y \partial / \partial y, y X_{i}, y^{2} Z_{j}$ lies in fact that the coefficients, on the right-hand side of (2.4), are independent of $y$. Also, for the hyperbolic space, (2.4) becomes

$$
\begin{aligned}
& \nabla_{y \partial / \partial y}\left(y \frac{\partial}{\partial y}\right)=0, \\
& \nabla_{y X_{i}}\left(y \frac{\partial}{\partial y}\right)-\nabla_{y \partial / \partial y}\left(y X_{i}\right)=\left[y X_{i}, y \frac{\partial}{\partial y}\right]=-y X_{i}, \\
& \nabla_{y \partial / \partial y}\left(y X_{i}\right)=0, \\
& \nabla_{y X_{i}}\left(y X_{j}\right)=\delta_{i j} y \frac{\partial}{\partial y} .
\end{aligned}
$$

Returning to the local expression for the tension field, we choose the frame field $e_{i}$ on $M$ to consist of $e_{0}=\partial / \partial y ; e_{i}=X_{i}, 1 \leq i \leq n_{1} ; e_{i}=Z_{i-n_{1}}$, $n_{1}+1 \leq i \leq n_{1}+n_{2}$. Similarly, on $\bar{M}$ it is natural to select $f_{0}=\partial / \partial \bar{y}$; $f_{\alpha}=\bar{X}_{\alpha}, 1 \leq i \leq \bar{n}_{1} ; f_{\alpha}=\bar{Z}_{\alpha-\bar{n}_{1}}, \bar{n}_{1}+1 \leq \alpha \leq \bar{n}_{1}+\bar{n}_{2}$. Using (2.1) and (2.3), we compute

$$
\begin{aligned}
& \tau^{0}(h)= g^{j j} h_{j j}^{0}+\left(1-n_{1}-2 n_{2}\right) h_{0}^{0} y-g^{j j} h_{j}^{0} h_{j}^{0} \bar{h}^{-1} \\
&+g^{j j} \sum_{\gamma=1}^{\bar{n}_{1}} h_{j}^{\gamma} h_{j}^{\gamma} \bar{y}^{-1}+g^{j j} \sum_{\gamma=\bar{n}_{1}+1}^{\bar{n}_{1}+\bar{n}_{2}} h_{j}^{\gamma} h_{j}^{\gamma}\left(2 \bar{y}^{-3}\right), \\
& \tau^{\alpha}(h)= g^{j j} h_{j j}^{\alpha}+\left(1-n_{1}-2 n_{2}\right) h_{0}^{\alpha} y-2 g^{j j} h_{j}^{0} h_{j}^{\alpha} \bar{y}^{-1} \\
&+g^{j j} \sum_{\beta=1}^{\bar{n}_{1}} \sum_{\gamma=\bar{n}_{1}+1}^{\bar{n}_{1}+\bar{n}_{2}} \alpha_{\alpha \beta}^{\gamma-\bar{n}_{1}} h_{j}^{\beta} h_{j}^{\gamma} \bar{y}^{-2}, \quad 1 \leq \alpha \leq \bar{n}_{1}, \\
& \tau^{\alpha}(h)= g^{j j} h_{j j}^{\alpha}+\left(1-n_{1}-2 n_{2}\right) h_{0}^{\alpha} y-4 g^{j j} h_{j}^{0} h_{j}^{\alpha} \bar{y}^{-1}, \\
& \bar{n}_{1}+1 \leq \alpha \leq \bar{n}_{1}+\bar{n}_{2} .
\end{aligned}
$$

Here $j$ is summed from 0 to $n_{1}+n_{2}$. Note that $\operatorname{dim} M=n_{1}+n_{2}+1$. If the domain $M$ is hyperbolic space, the formulas (2.5) hold with $n_{2}=0$. For $\bar{M}$ 
of constant negative curvature, we have the analogous formulas

$$
\begin{aligned}
\tau^{0}= & g^{j j} h_{j j}^{0}+\left(1-n_{1}-2 n_{2}\right) h_{0}^{0} y-g^{j j} h_{j}^{0} h_{j}^{0} \bar{y}^{-1} \\
& +g^{j j} \sum_{\gamma=1}^{\bar{n}_{1}} h_{j}^{\gamma} h_{j}^{\gamma} \bar{y}^{-1}, \\
\tau^{\alpha}(h)= & g^{j j} h_{j j}^{\alpha}+\left(1-n_{1}-2 n_{2}\right) h_{0}^{\alpha} y-2 g^{j j} h_{j}^{0} h_{j}^{\alpha} \bar{y}^{-1}, \quad 1 \leq \alpha \leq \bar{n}_{1} .
\end{aligned}
$$

\section{NECESSARY CONDITIONS AND UNIQUENESS}

Suppose that $M$ is a simply connected, rank one, symmetric space of noncompact type. The exponential map, from any basepoint, provides a diffeomorphism between $M$ and a Euclidean space with the dimension of $M$. One compactifies $M$ by adding a sphere at infinity. The compactification $M^{\prime}$ of $M$ is thus homeomorphic to a Euclidean ball of the same dimension as $M$. Moreover, this compactification $M^{\prime}$ admits the structure of a $C^{\infty}$ manifold with boundary. The boundary coordinate charts are given by the Cayley transform. In such charts, the metric admits the representation (2.2), with the ideal boundary portion contained in $0 \times N$.

Let $h: M \rightarrow \bar{M}$ be a $C^{2}$ proper map between rank one symmetric spaces of noncompact type. Suppose that $h$ extends to a $C^{1}$ map $h: M^{\prime} \rightarrow \bar{M}^{\prime}$ from the compactification $M^{\prime}$ of $M$, to the compactification $\bar{M}^{\prime}$ of $\bar{M}$. We plan to investigate necessary conditions satisfied by the first derivatives of $h$ at, the boundary, when $h$ is harmonic in the interior $M$. We begin with some preparatory lemmas:

Lemma 3.1. Assume that $V_{j}$ are $n$ linearly independent $C^{\infty}$ vector fields defined on a ball, centered at $p$, in $n$-dimensional Euclidean space. Given real numbers $\alpha_{j}$, there exists a $C^{\infty}$ function $\psi$ so that $V_{j} \psi(p)=\alpha_{j}$ and $V_{j} V_{j} \psi(p)=0$, for each fixed $j=1,2, \ldots, n$.

Proof. If $x_{k}$ are local coordinates, then we may write $V_{j}=\sum_{k} a_{j k}(x)\left(\partial / \partial x_{k}\right)$, where $a_{j k}$ is an invertible matrix. The first derivatives of $\psi$ are determined by $\sum_{k} a_{j k}(p) \partial \psi(p) / \partial x_{k}=\alpha_{j}$, that is $\partial \psi(p) / \partial x_{k}=\sum a_{k s}^{-1}(p) \alpha_{s}$.

For the conditions on the second derivatives, one has

$$
\begin{aligned}
& 0=V_{j} V_{j} \psi(p)=\sum_{k} a_{j k} \frac{\partial}{\partial x_{k}} \sum_{s} a_{j s} \frac{\partial}{\partial x_{s}} \psi, \\
& 0=\sum_{k, s} a_{j k} a_{j s} \frac{\partial^{2} \psi}{\partial x_{k} \partial x_{s}}+\sum_{k} a_{j k} \frac{\partial a_{j s}}{\partial x_{k}} \frac{\partial \psi}{\partial x_{s}} .
\end{aligned}
$$

Define

$$
\beta_{j}=-\sum_{k} a_{j k} \frac{\partial a_{j s}}{\partial x_{k}} \frac{\partial \psi}{\partial x_{s}},
$$

evaluated at $p$. Let $b$ denote a diagonal matrix with entries $b_{j j}=\beta_{j}$. The condition $V_{j} V_{j} g(p)=0$ may be written as $\left(a(\operatorname{Hess} \psi) a^{t}\right)_{j j}=b_{j j}$, where $a^{t}$ is the transpose of $a$. It suffices to choose Hess $\psi=a^{-1} b\left(a^{t}\right)^{-1}$, a symmetric matrix.

We apply the preceding lemma in a coordinate chart centered at a boundary point $p$ of the compactification $M^{\prime}$ of $M$. In the unbounded model the 
metric is given by (2.2) and we may choose $p=(0, e) \in R \times N$, where $e$ is the identity element in the group $N$. The collection of vector fields $V_{j}=e_{j}$ consists of $\partial / \partial y, X_{k}, Z_{l}$, with $1 \leq k \leq n_{1}, n_{1}+1 \leq l \leq n_{1}+n_{2}$, and $0 \leq j \leq n_{1}+n_{2}$. The Laplacian of $M$, acting on functions, has the form

$$
\Delta \psi=\sum_{j} g^{j j} e_{j} e_{j} \psi+\left(1-n_{1}-2 n_{2}\right) y \frac{\partial \psi}{\partial y} .
$$

More generally, if $\phi=\sum_{i} \phi_{i} e_{i}^{*}$ is a 1 -form, then the divergence of $\phi$ is given by

$$
\delta \phi=\sum_{j} g^{j j} e_{j} \phi_{j}+\left(1-n_{1}-2 n_{2}\right) y \phi_{0} .
$$

If $\phi=d \psi$, then $\Delta \psi=\delta d \psi=\delta \phi$. Under the circumstances, one has

Lemma 3.2. Suppose that $\phi \in C^{1} \Lambda^{1} M \cap C^{0} \Lambda^{1} M^{\prime}$, is a 1-form defined on a neighborhood of $p \in M^{\prime}$. If $\phi=\sum_{i} \phi_{i} e_{i}^{*}$, then there is a sequence of points $q_{k} \rightarrow p$, with $\sum_{j} g^{j j}\left(e_{j} \phi_{j}\right) y^{-1} \rightarrow 0$.

Proof. If $\phi \in C^{1} \Lambda^{1} M^{\prime}$, the conclusion holds for any sequence converging to $p$, since $g^{j j}=O\left(y^{2}\right), 0 \leq j \leq n_{1}+n_{2}$. Under the weaker hypothesis of the lemma, $\psi \in C^{1} \Lambda^{1} M \cap C^{0} \Lambda^{1} M^{\prime}$, more argument is required. By Lemma 3.1, we may choose a $C^{\infty}$ function $\psi$ with $d \psi(p)=\phi(p)$ and $e_{j} e_{j} \psi(p)=0$, for all $0 \leq j \leq n_{1}+n_{2}$. let $p_{k} \rightarrow p$ be any sequence and use the symbol $B\left(p_{k}, 1\right)$ to denote the unit ball centered at $p$, relative to the complete metric (2.2).

By Stokes' theorem,

$$
\int_{B\left(p_{k}, 1\right)} \delta \phi=\int_{\partial B\left(p_{k}, 1\right)} \phi(\nu)=\int_{\partial B\left(p_{k}, 1\right)} d \psi(\nu)+\varepsilon_{1} y
$$

where $\nu$ is a unit outward normal to $\partial B\left(p_{k}, 1\right)$. The symbols $\varepsilon_{i}$ will denote quantities which become arbitrarily small as $p_{k} \rightarrow p$. The factor $y$ appears because we measure the length of covectors in the invariant metric of the symmetric space.

Applying Stokes' theorem again,

$$
\int_{B\left(p_{k}, 1\right)} \delta \phi=\int_{B\left(p_{k}, 1\right)} \Delta \psi+\varepsilon_{1} y
$$

By Lemma 3.1,

$$
\Delta \psi=\left(1-n_{1}-2 n_{2}\right) \phi\left(\frac{\partial}{\partial y}\right)+\varepsilon_{2} y .
$$

Consequently, by the formula for $\delta \phi$ given above,

$$
\frac{1}{y} \int_{B\left(p_{k}, 1\right)} \sum_{j} g^{j j} e_{j} \phi_{j} \rightarrow 0, \quad \text { as } p_{k} \rightarrow p .
$$

Since the balls $B\left(p_{k}, 1\right)$ have volume independent of $k$, there exists a sequence $q_{k} \in B\left(p_{k}, 1\right)$ satisfying the conclusion of Lemma 3.2.

We return to our map $h \in C^{2}(M, \bar{M}) \cap C^{1}\left(M^{\prime}, \bar{M}^{\prime}\right)$. Formula (2.5) gives the $\partial / \partial y$ component of the tension of $h$. If $\tau^{0}(h)=O\left(y^{-1+\varepsilon}\right)$, then multiply the formula, $(2.5)$, for $\tau^{0}(h)$ by $\bar{y}^{3} y^{-2}$, and let $y \rightarrow 0$, to deduce 
Condition 3.3. $\sum_{j=0}^{n_{1}} \sum_{\gamma=\bar{n}_{1}+1}^{\bar{n}_{1}+\bar{n}_{2}} h_{j}^{\gamma} h_{j}^{\gamma}=0$, at the boundary.

Here, we applied Lemma 3.2 to $\phi=\sum h_{j}^{0} e_{j}^{*}$, in order to eliminate the terms involving second derivatives. In particular, a notable special case is

Proposition 3.4. Suppose that $h: M \rightarrow \bar{M}$ is $C^{2}$ proper harrnonic map which extends to a $C^{1}$ map $h: M^{\prime} \rightarrow \bar{M}^{\prime}$. Let $f: \partial M^{\prime} \rightarrow \partial M^{\prime}$ be the boundary values of $h$. Then $\sum_{j=1}^{n_{1}} \sum_{\gamma=\bar{n}_{1}+1}^{\bar{n}_{1}+\bar{n}_{2}} f_{j}^{\gamma} f_{j}^{\gamma}=0$.

If $h$ is a harmonic self-map of the rank one Hermitian space, the unit ball in $C^{n}$ with its Bergman metric, then Proposition 3.4 states that the boundary value of $h$ is a contact transformation. This example is typical of the situation where the range is a rank one symmetric space, but not the hyperbolic space.

If $\bar{M}$ is hyperbolic, then Condition 3.3 is vacuous, and we now consider this situation. Suppose $h \in C^{2}(M, \bar{M}) \cap C^{1}\left(M^{\prime}, \bar{M}^{\prime}\right)$ and $\bar{M}$ has constant negative curvature. The formulas (2.5a) are now applicable. If $\tau^{\alpha}(h)=O\left(y^{1+\varepsilon}\right), \alpha \geq 0$, for some $\varepsilon>0$, then multiply (2.5a) by $\bar{y} y^{-2}$ and let $y \rightarrow 0$ to deduce

$$
\begin{aligned}
& \left(-n_{1}-2 n_{2}\right)\left(h_{0}^{0}\right)^{2}+\sum_{j=0}^{n_{1}} \sum_{\gamma=1}^{n_{1}} h_{j}^{\gamma} h_{j}^{\gamma}=0, \\
& \left(-1-n_{1}-2 n_{2}\right) h_{0}^{\alpha} h_{0}^{0}=0, \quad \alpha \geq 1 .
\end{aligned}
$$

Again, we employed Lemma 3.2 to handle the second order terms. Also, note that $h_{j}^{0}=0$ at the boundary, for $j \geq 1$, since $h: \partial M^{\prime} \rightarrow \partial \bar{M}^{\prime}$. It is easy to deduce

Condition 3.3a. If the range $\bar{M}$ has constant negative curvature and the boundary values $f$ satisfy $\sum_{j=1}^{n_{1}} \sum_{\gamma=1}^{\bar{n}_{1}} f_{j}^{\gamma} f_{j}^{\gamma}>0$, then, at the boundary,

$$
h_{0}^{0}=\left[\sum_{j=1}^{n_{1}} \sum_{\gamma=1}^{\bar{n}_{1}} \frac{f_{j}^{\gamma} f_{j}^{\gamma}}{n_{1}+2 n_{2}}\right]^{1 / 2} ; \quad h_{0}^{\alpha}=0, \alpha \geq 1 .
$$

This leads to the following uniqueness theorem.

Proposition 3.5. Let $h$ and $\hat{h}$ be proper harmonic maps from the rank one symmetric space $M$ to the hyperbolic space $\bar{M}$, of constant negative curvature. Assume that both $h$ and $\hat{h}$ extend to maps in $C^{1}\left(M^{\prime}, \bar{M}^{\prime}\right)$. If $h, \hat{h}$ have the same boundary map $f: \partial M^{\prime} \rightarrow \partial \bar{M}$, and $\sum_{j=1}^{n_{1}} \sum_{\gamma=1}^{\bar{n}_{1}} f_{j}^{\gamma} f_{j}^{\gamma}>0$ then $h$ and $\hat{h}$ are identical.

Proof. If both $M$ and $\bar{M}$ are hyperbolic, this was proved by Li and Tam [8]. Their proof extends with only minor changes.

Consider again a proper map between arbitrary rank one symmetric spaces $M, \bar{M}$ of noncompact type. To proceed further, we make the additional assumption that our map $h$ is $C^{2}$ up to the boundary of the compactification. If $\tau^{0}(h)=O\left(y^{1+\varepsilon}\right)$, then Condition 3.3 holds, and we may multiply (2.5) by $\bar{y}^{3} y^{-4}$, letting $y \rightarrow 0$ to give 


\section{Condition 3.6.}

$$
\begin{aligned}
\left(n_{1}+2 n_{2}\right)\left(h_{0}^{0}\right)^{4} & -\sum_{j=0}^{n_{1}} \sum_{\gamma=1}^{\bar{n}_{1}} h_{j}^{\gamma} h_{j}^{\gamma}\left(h_{0}^{0}\right)^{2}-2 \sum_{j=0}^{n_{1}} \sum_{\gamma=\bar{n}_{1}+1}^{\bar{n}_{1}+\bar{n}_{2}} h_{j 0}^{\gamma} h_{j 0}^{\gamma} \\
& -2 \sum_{j=n_{1}+1}^{n_{1}+n_{2}} \sum_{\gamma=\bar{n}_{1}+1}^{\bar{n}_{1}+\bar{n}_{2}} h_{j}^{\gamma} h_{j}^{\gamma}=0,
\end{aligned}
$$

at the boundary.

Similarly, for $1 \leq \alpha \leq \bar{n}_{1}$, we suppose that $\tau^{\alpha}(h)=O\left(y^{1+\varepsilon}\right)$. Multiplying (2.5) by $\bar{y}^{2} y^{-3}$ and letting $y \rightarrow 0$ yields, assuming our previously established Condition 3.3:

\section{Condition 3.7.}

$$
\left(1+n_{2}+2 n_{2}\right) h_{0}^{\alpha}\left(h_{0}^{0}\right)^{2}-\sum_{j=0}^{n_{1}} \sum_{\beta=1}^{\bar{n}_{1}} \sum_{\gamma=\bar{n}_{1}+1}^{\bar{n}_{1}+\bar{n}_{2}} \alpha_{\alpha \beta}^{\gamma-\bar{n}_{1}} h_{j}^{\beta} h_{j 0}^{\gamma}=0,
$$

at the boundary.

Finally, we consider $\bar{n}_{1}+1 \leq \alpha \leq \bar{n}_{1}+\bar{n}_{2}$ and suppose that $\tau^{\alpha}(h)=O\left(y^{2+\varepsilon}\right)$. Multiplying (2.5) by $\bar{y} y^{-3}$, using Condition 3.3, and letting $y \rightarrow 0$, gives

Condition 3.8. $\left(2+n_{1}+2 n_{2}\right) h_{0}^{0} h_{00}^{\alpha}=0$, at the boundary.

Note that $h_{j}^{\alpha}=0$, along the boundary, by condition 3.3 , for $1 \leq j \leq n_{1}$, $\bar{n}_{1}+1 \leq \alpha \leq \bar{n}_{1}+\bar{n}_{2}$. Since $e_{j}$ is tangent to the boundary, we also have $h_{j j}^{\alpha}=e_{j} h_{j}^{\alpha}=0$, for such $j$ and $\alpha$.

To proceed further, we consider the integrability condition $d d h=0$, which holds for any $C^{2}$ map $h$ [2]. Recall that $d h=h_{i}^{\alpha} e_{i}^{*} \otimes f_{\alpha}$, and

$$
\nabla d h=h_{i j}^{\alpha} e_{j}^{*} \otimes e_{i}^{*} \otimes f_{\alpha}+h_{i}^{\alpha} e_{j}^{*} \otimes \nabla_{e_{j}} e_{i}^{*} \otimes f_{\alpha}+h_{i}^{\alpha} e_{j}^{*} \otimes e_{i}^{*} \otimes \nabla_{e_{j}} f_{\alpha} .
$$

Thus

$$
0=d d h=h_{i j}^{\alpha} e_{j}^{*} \wedge e_{i}^{*} \otimes f_{\alpha}+h_{i}^{\alpha} e_{j}^{*} \wedge \nabla_{e_{j}} e_{i}^{*} \otimes f_{\alpha}+h_{i}^{\alpha} e_{j}^{*} \wedge e_{i}^{*} \otimes \nabla_{e_{j}} f_{\alpha} .
$$

However, $\nabla_{e_{j}} e_{i}^{*}=e_{k}\left(\nabla_{e_{j}} e_{i}^{*}\right) e_{k}^{*}=-e_{i}^{*}\left(\nabla_{e_{j}} e_{k}\right) e_{k}^{*}$ and thus

$$
h_{i j}^{\alpha} e_{j}^{*} \wedge e_{i}^{*} \otimes f_{\alpha}-h_{i}^{\alpha} e_{i}^{*}\left(\nabla_{e_{j}} e_{k}\right) e_{j}^{*} \wedge e_{k}^{*} \otimes f_{\alpha}+h_{i}^{\gamma} h_{j}^{\beta} e_{j}^{*} \wedge e_{i}^{*} \otimes \nabla_{f_{\beta}} f_{\gamma}=0 .
$$

This may be rewritten as

$$
h_{i j}^{\alpha} e_{j}^{*} \wedge e_{i}^{*} \otimes f_{\alpha}-\frac{1}{2} h_{i}^{\alpha} e_{i}^{*}\left(\left[e_{j}, e_{k}\right]\right) e_{j}^{*} \wedge e_{k}^{*} \otimes f_{\alpha}+\frac{1}{2} h_{i}^{\gamma} h_{j}^{\beta} e_{j}^{*} \wedge e_{i}^{*} \otimes\left[f_{\beta}, f_{\gamma}\right]=0
$$

or equivalently

$$
h_{i j}^{\alpha} e_{i}^{*} \wedge e_{j}^{*}=-\frac{1}{2} h_{i}^{\alpha} e_{i}^{*}\left(\left[e_{j}, e_{k}\right]\right) e_{j}^{*} \wedge e_{k}^{*}+\frac{1}{2} h_{i}^{\beta} h_{j}^{\gamma} f_{\alpha}^{*}\left(\left[f_{\beta}, f_{\gamma}\right]\right) e_{i}^{*} \wedge e_{j}^{*} .
$$

Note that the expression $h_{k 0}^{\alpha}$ for $1 \leq k \leq n_{1}, \bar{n}_{1}+1 \leq \alpha \leq \bar{n}_{1}+\bar{n}_{2}$, occurs in the Condition 3.7. By Condition 3.3, one has $h_{0 k}^{\alpha}=0$, since $e_{k}$ is tangent to the boundary. So, we may deduce from (3.9) that

$$
h_{k 0}^{\alpha}=\frac{1}{2}\left(h_{k}^{\beta} h_{0}^{\gamma}-h_{0}^{\beta} h_{k}^{\gamma}\right) f_{\alpha}^{*}\left(\left[f_{\beta}, f_{\gamma}\right]\right)=\sum_{\beta=1}^{\bar{n}_{1}} \sum_{\gamma=1}^{\bar{n}_{1}} h_{k}^{\beta} h_{0}^{\gamma} a_{\beta \gamma}^{\alpha-\bar{n}_{1}} .
$$

Relabeling the indices and substituting back into Condition 3.7 gives $1 \leq$ $\alpha \leq \bar{n}_{1}$, 
Condition 3.11.

$$
\left(1+n_{1}+2 n_{2}\right) h_{0}^{\alpha}\left(h_{0}^{0}\right)^{2}+\sum_{j=0}^{\bar{n}_{1}} \sum_{\beta=1}^{\bar{n}_{1}} \sum_{\varepsilon=1}^{\bar{n}_{1}} \sum_{\mu=1}^{\bar{n}_{1}} \sum_{\gamma=\bar{n}_{1}+1}^{\bar{n}_{1}+\bar{n}_{2}} \alpha_{\beta \alpha}^{\gamma-\bar{n}_{1}} h_{j}^{\beta} h_{j}^{\varepsilon} \alpha_{\varepsilon \mu}^{\gamma-\bar{n}_{1}} h_{0}^{\mu}=0
$$

at the boundary.

In preparation for our uniqueness theorem, we deduce

Lemma 3.12. Let $h \in C^{2}\left(M^{\prime}, \bar{M}^{\prime}\right)$ satisfy $3.3,3.6,3.7,3.8$ on $M$. Assume that the boundary data $f$ satisfies $\sum_{j=n_{1}+1}^{n_{1}+n_{2}} \sum_{\gamma=\bar{n}_{1}+1}^{\bar{n}_{1}+\bar{n}_{2}} f_{j}^{\gamma} f_{j}^{\gamma}>0$ then $h_{0}^{0}>0, h_{0}^{\alpha}=0$ for $1 \leq \alpha \leq \bar{n}_{1}+\bar{n}_{2}$, and $h_{k 0}^{\beta}=h_{00}^{\beta}=0$ for $\bar{n}_{1}+1 \leq \beta \leq \bar{n}_{1}+\bar{n}_{2}, 1 \leq k \leq n_{1}$. Proof. The inequality $h_{0}^{0}>0$ follows immediately from Condition 3.6 and our hypothesis about the boundary data. If $\bar{n}_{1}+1 \leq \alpha \leq \bar{n}_{1}+\bar{n}_{2}$, then $h_{0}^{\alpha}=0$, by Condition 3.3 .

If $1 \leq \alpha \leq \bar{n}_{1}$, then we multiply Condition 3.11 by $h_{0}^{\alpha}$ and sum over $\alpha$, yielding

$$
\left(1+n_{1}+2 n_{2}\right)\left(h_{0}^{0}\right)^{2} \sum_{\alpha=1}^{\bar{n}_{1}}\left(h_{0}^{\alpha}\right)^{2}+\sum_{\gamma=\bar{n}_{1}+1}^{\bar{n}_{1}+\bar{n}_{2}} \sum_{j=0}^{\bar{n}_{1}}\left(\sum_{\varepsilon, \mu=1}^{\bar{n}_{1}} h_{j}^{\varepsilon} a_{\varepsilon \mu}^{\gamma-\bar{n}_{1}} h_{0}^{\mu}\right)^{2}=0 .
$$

Since all terms in the sum are positive, $h_{0}^{\alpha}=0$.

It now follows from (3.10) that $h_{k 0}^{\beta}=0$, for $1 \leq k \leq n_{1}$ and $\bar{n}_{1}+1 \leq \beta \leq$ $\bar{n}_{1}+\bar{n}_{2}$, since we have just shown that $h_{0}^{\gamma}=0$, for $1 \leq \gamma \leq \bar{n}_{1}$. Condition 3.8 implies $h_{00}^{\beta}=0$, when $\bar{n}_{1}+1 \leq \beta \leq \bar{n}_{1}+\bar{n}_{2}$.

This allows us to derive the following uniqueness result.

Theorem 3.13. Let $h$ and $\hat{h}$ be proper harmonic maps between rank one symmetric spaces of noncompact type. Assume that both $h$ and $\hat{h}$ extend to maps in $C^{2}\left(M^{\prime}, \bar{M}^{\prime}\right)$. If $h, \hat{h}$ both have the same boundary map $f: \partial M^{\prime} \rightarrow \partial \bar{M}^{\prime}$ and $\sum_{j=n_{1}+1}^{n_{1}+n_{2}} \sum_{\gamma=\bar{n}_{1}+1}^{\bar{n}_{1}+\bar{n}_{2}} f_{j}^{\gamma} f_{j}^{\gamma}>0$ then $h=\hat{h}$, everywhere.

Proof. Let $(y, n) \in R^{+} \times N$ and $(\bar{y}, \bar{n}) \in R^{+} \times \bar{N}$ be local coordinates near the boundary of $M^{\prime}$ and $\bar{M}^{\prime}$. If $d$ denotes the Riemannian distance in $\bar{M}$, then

$$
\begin{aligned}
d(h, \hat{h}) \leq & d(h,(\bar{y}(h), \bar{n}(f)))+d((\bar{y}(h), \bar{n}(f)),(\bar{y}(\hat{h}), \bar{n}(f))) \\
& +d((\bar{y}(\hat{h}), \bar{n}(f)), \hat{h}) .
\end{aligned}
$$

To estimate the first term, we consider the curve $(\bar{y}(h), \bar{n}(h(t, n))), 0 \leq t \leq$ $y$, which joins $(\bar{y}(h), \bar{n}(f))$ to $h=h(y, n)$. One has

$$
d(h,(\bar{y}(h), \bar{n}(f))) \leq c_{1} \int_{0}^{y}\left(\bar{y}^{-1} \sum_{\alpha=1}^{\bar{n}_{1}}\left|h_{0}^{\alpha}\right|+\bar{y}^{-2} \sum_{\alpha=\bar{n}_{1}+1}^{\bar{n}_{1}+\bar{n}_{2}}\left|h_{0}^{\alpha}\right|\right) d t .
$$

By Lemma 3.12, $\left|h_{0}^{\alpha}\right|=O(t)$, for $1 \leq \alpha \leq \bar{n}_{1}+\bar{n}_{2} ;\left|h_{0}^{\alpha}\right|=o(t)$, for $\bar{n}_{1}+1 \leq$ $\alpha \leq \bar{n}_{1}+\bar{n}_{2}$. Since $h_{0}^{0}>0, y$ is comparable to $\bar{y}$. So

$$
d(h,(\bar{y}(h), \bar{n}(f))) \leq c_{2}\left(y^{-1} \int_{0}^{y} t d t+y^{-2} \int_{0}^{y} o(t) d t\right)
$$


So $d(h,(\bar{y}(h), \bar{n}(f)))=o(1)$. The third term is completely analogous.

To estimate the second term, we employ the curve $(t, \bar{n}(f)), \bar{y}(h) \leq t \leq$ $\bar{y}(\hat{h})$. Condition 3.6 implies that $h_{0}^{0}=\hat{h}_{0}^{0}$. This is because Lemma 3.12 implies $h_{j 0}^{\gamma}=\hat{h}_{j 0}^{\gamma}=0,0 \leq j \leq n_{1}, \bar{n}_{1}+1 \leq \gamma \leq \bar{n}_{1}+\bar{n}_{2}$, and for $1 \leq \alpha \leq \bar{n}_{1}$, $h_{0}^{\alpha}=\hat{h}_{0}^{\alpha}=0$. So

$$
\begin{aligned}
d((\bar{y}(h), \bar{n}(f)),(\bar{y}(\hat{h}), \bar{n}(f))) & =\left|\int_{\bar{y}(h)}^{\bar{y}(\hat{h})} \frac{d t}{t}\right|=\left|\ln \left(\frac{\bar{y}(\hat{h})}{\bar{y}(h)}\right)\right| \\
& =\left|\ln \left(\frac{h_{0}^{0} y+o(y)}{h_{0}^{0} y+o(y)}\right)\right|=o(1) .
\end{aligned}
$$

Thus $d^{2}(h, \hat{h})$ is a subharmonic function, which vanishes on $\partial M^{\prime}$. it must be identically zero. The subharmonicity is well known, since the range has nonpositive curvature [10].

\section{EXISTENCE}

The necessary conditions of the previous section lead naturally to a construction of harmonic maps, given sufficiently regular boundary data. Assume that $M$ and $\bar{M}$ are two rank one symmetric spaces of noncompact type, with their compactifications $M^{\prime}$ and $\bar{M}^{\prime}$. Let $f: \partial M^{\prime} \rightarrow \partial \bar{M}^{\prime}$ be a $C^{2, \varepsilon}$ map, $0<\varepsilon<1$, satisfying $f_{j}^{\gamma}=0,1 \leq j \leq n_{1}, \bar{n}_{1}+1 \leq \gamma \leq \bar{n}_{1}+\bar{n}_{2}$. Our goal is to construct a harmonic map $M \rightarrow \bar{M}$, which assumes the boundary values $f$. This will be achieved by constructing a map $h$, with boundary data $f$, whose tension field decays to zero at infinity, and then applying the nonlinear heat equation to deform our approximate solution to a harmonic map.

Our first step is to establish a converse to the necessary conditions of $\S 3$.

Lemma 4.1. Let $h \in C^{2, \varepsilon}\left(M^{\prime}, \bar{M}^{\prime}\right), 0<\varepsilon<1$. Such $h$ satisfies the following conditions, at the boundary

(i)

(ii)

$$
\sum_{j=0}^{n_{1}} \sum_{\gamma=\bar{n}_{1}+1}^{\bar{n}_{1}+\bar{n}_{2}} h_{j}^{\gamma} h_{j}^{\gamma}=0
$$

$$
\begin{aligned}
\left(n_{1}+2 n_{2}\right)\left(h_{0}^{0}\right)^{4} & -\sum_{j=0}^{n_{1}} \sum_{\gamma=1}^{\bar{n}_{1}} h_{j}^{\gamma} h_{j}^{\gamma}\left(h_{0}^{0}\right)^{2} \\
& -2 \sum_{j=0}^{n_{1}} \sum_{\gamma=\bar{n}_{1}+1}^{\bar{n}_{1}+\bar{n}_{2}} h_{j 0}^{\gamma} h_{j 0}^{\gamma}-2 \sum_{j=n_{1}+1}^{n_{1}+n_{2}} \sum_{\gamma=\bar{n}_{1}+1}^{\bar{n}_{1}+\bar{n}_{2}} h_{j}^{\gamma} h_{j}^{\gamma}=0,
\end{aligned}
$$

(iii)

$$
\left(1+n_{1}+2 n_{2}\right) h_{0}^{\alpha}\left(h_{0}^{0}\right)^{2}-\sum_{j=0}^{n_{1}} \sum_{\beta=1}^{\bar{n}_{1}} \sum_{\gamma=\bar{n}_{1}+1}^{\bar{n}_{1}+\bar{n}_{2}} a_{\alpha \beta}^{\gamma-\bar{n}_{1}} h_{j}^{\beta} h_{j 0}^{\gamma}=0, \quad 1 \leq \alpha \leq \bar{n}_{1},
$$

$$
h_{0}^{0} h_{00}^{\alpha}=0, \quad \bar{n}_{1}+1 \leq \alpha \leq \bar{n}_{1}+\bar{n}_{2},
$$


whenever $\tau^{0}(h)=O\left(y^{1+\varepsilon}\right) ; \tau^{\alpha}(h)=O\left(y^{1+\varepsilon}\right), 1 \leq \alpha \leq \bar{n}_{1} ; \tau^{\alpha}(h)=O\left(y^{2+\varepsilon}\right)$, $\bar{n}_{1}+1 \leq \alpha \leq \bar{n}_{1}+\bar{n}_{2}$. Conversely, if conditions (i)-(iv) hold, then the components $\tau^{\alpha}(h)$, of the tension field, have the indicated decay as $y \downarrow 0$, provided $h_{0}^{0}>0$.

Proof. In $\S 3$, we established (i)-(iv), for any $h \in C^{2}\left(M^{\prime}, \bar{M}^{\prime}\right)$, whose tension field decays as supposed. The converse assertion follows from (2.5). If $h_{0}^{0}>0$, then the second order Taylor expansion, of $h$, gives corresponding approximations for the components of $\tau(h)$. Conditions (i) and (ii) force the vanishing of the first two terms approximating $\tau^{0}(h)$, the remainder is of order $y^{1+\varepsilon}$. Conditions (iii), (i) imply that the lead two terms for $\tau^{\alpha}(h), 1 \leq \alpha \leq \bar{n}_{1}$, are zero, so $\tau^{\alpha}(h)=O\left(y^{1+\varepsilon}\right)$. Lastly, conditions (iv), (i) imply that the first two terms for $\tau^{\alpha}(h), \bar{n}_{1}+1 \leq \alpha \leq \bar{n}_{1}+\bar{n}_{2}$, are zero, so $\tau^{\alpha}(h)=O\left(y^{2+\varepsilon}\right)$.

Next, we construct an asymptotically harmonic map, with appropriately given boundary values:

Proposition 4.2. Suppose that $f \in C^{2, \varepsilon}\left(\partial M^{\prime}, \partial \bar{M}^{\prime}\right), 0<\varepsilon<1$, satisfies $f_{j}^{\gamma}=0,1 \leq j \leq n_{1}, \bar{n}_{1}+1 \leq \gamma \leq \bar{n}_{1}+\bar{n}_{2}$, and $\sum_{j=n_{1}+1}^{n_{1}+n_{2}} \sum_{\gamma=\bar{n}_{1}+1}^{\bar{n}_{1}+\bar{n}_{2}} f_{j}^{\gamma} f_{j}^{\gamma}>0$. Then there exists $h \in C^{2, \varepsilon}\left(M^{\prime}, \bar{M}^{\prime}\right)$, assuming the boundary values $f$ continuously, with $\|\tau(h)\|=O\left(y^{\varepsilon}\right)$. Here $\|\tau(h)\|$ is the norm of the tension field in the Riemannian norm.

Proof. Motivated by (ii) of Lemma 4.1, we let $\phi>0$ be a solution of

$$
\left(n_{1}+2 n_{2}\right) \phi^{4}-\sum_{j=1}^{n_{1}} \sum_{\gamma=1}^{\bar{n}_{1}} f_{j}^{\gamma} f_{j}^{\gamma} \phi^{2}-2 \sum_{j=n_{1}+1}^{n_{1}+n_{2}} \sum_{\gamma=\bar{n}_{1}+1}^{\bar{n}_{1}+\bar{n}_{2}} f_{j}^{\gamma} f_{j}^{\gamma}=0 .
$$

In our local chart near the boundary, we extend $\phi$, by convolving with a smoothing kernel, commensurable to the Euclidean Poisson kernel. Since $f \in C^{2, \varepsilon}, \phi$ and its extension lies in $C^{1, \varepsilon}$, moreover [11], $\left|\nabla_{0}^{2} \phi\right|=O\left(y^{\varepsilon-1}\right)$, by an elementary Poisson kernel estimate. Here $\left|\nabla_{0}^{2} \phi\right|$ is a locally defined Euclidean norm, in our chart. Define $h(y, n)=(y \phi(y, n), f(n))$. Then $h \in C^{2, \varepsilon}$.

For this $h, h_{0}^{0}=\phi ; h_{0}^{\alpha}=0,1 \leq \alpha \leq \bar{n}_{1}+\bar{n}_{2}, h_{00}^{\alpha}=0, \bar{n}_{1}+1 \leq \alpha \leq \bar{n}_{1}+\bar{n}_{2}$; $h_{j 0}^{\gamma}=0,1 \leq j \leq n_{1}, \bar{n}_{1}+1 \leq \gamma \leq \bar{n}_{1}+\bar{n}_{2}$, and $h$ restricts to $f$ on $\partial M^{\prime}$. Thus, conditions (i)-(iv) of Lemma 4.1 hold at the boundary. By Lemma 4.1 and the expression (2.2) of the metric $\|\tau(h)\|=O\left(y^{\varepsilon}\right)$. This completes the local construction. Since $\|\tau(h)\|=O\left(y^{\varepsilon}\right)$, and Lemma 4.1 is an equivalence statement, (i)-(iv) are valid in any coordinate patch. Thus, the conclusion of Lemma 3.12 holds, not just in the chart where $h$ was constructed, but in any overlapping chart. We now fit together our local solutions via partition of unity, along the boundary. The partition functions can be chosen independent of $y$, near $\partial \bar{M}^{\prime}$. The conclusion of Lemma 3.12 is seen to hold for our global solution. However, this implies (i)-(iv) of Lemma 4.1 and thus $\|\tau(h)\|=O\left(y^{\varepsilon}\right)$.

The deformation, via the nonlinear heat equation, employs certain superharmonic functions as barriers. In standard notation, let $r$ denote the geodesic distance from the basepoint in our rank one symmetric space $M$. One has

Lemma 4.3. Assume that $r_{0}$ is sufficiently large. Define, for any given $0<$ $s \leq n_{1}+2 n_{2}, \psi(r)=e^{-s r}, r \geq r_{0}$, and $\psi(r)=e^{-s r_{0}}, r \leq r_{0}$. Then $\psi$ is superharmonic, on $M$. 
Proof. In exponential polar coordinates $(r, w)$, the volume element is written as $(\sinh r)^{n_{1}}(\sinh 2 r)^{n_{2}} d r d w$. To check our normalization of metric, observe that $r$ is commensurable to $-\ln y$ in (2.2). If $r \geq r_{0}$, the standard expression for the Laplacian of a radial function gives

$$
\begin{aligned}
\Delta \psi(r) & =\psi^{\prime \prime}(r)+\left(n_{1} \frac{\cosh r}{\sinh r}+2 n_{2} \frac{\cosh 2 r}{\sinh 2 r}\right) \psi^{\prime}(r) \\
& =s\left(s-n_{1} \frac{\cosh r}{\sinh r}-2 n_{2} \frac{\cosh 2 r}{\sinh 2 r}\right) \psi(r) .
\end{aligned}
$$

So $\Delta \psi(r) \leq 0$, because $0<s \leq n_{1}+2 n_{2}$. Since the minimum of two superharmonic functions is superharmonic and superharmonic is a local concept, $\psi$ is superharmonic on all of $M$.

Combining Proposition 4.2, Lemma 4.3, and the method of Li and Tam [8], one deduces

Theorem 4.4. Suppose that $f \in C^{2, \varepsilon}\left(\partial M^{\prime}, \partial \bar{M}^{\prime}\right), 0<\varepsilon<1$, satisfies $f_{j}^{\gamma}=0$, $1 \leq j \leq n_{1}, \bar{n}_{1}+1 \leq \gamma \leq \bar{n}_{1}+\bar{n}_{2}$, and $\sum_{j=n_{1}+1}^{n_{1}+n_{2}} \sum_{\gamma=\bar{n}_{1}+1}^{\bar{n}_{1}+\bar{n}_{2}} f_{j}^{\gamma} f_{j}^{\gamma}>0$. Then there exists a harmonic map $u: M \rightarrow \bar{M}$, which assumes the boundary values $f$, continuously. If $h$ is the map of Proposition 4.2, then the Riemannian distance from $h$ to $u$ is $O\left(y^{\bar{\varepsilon}}\right)$, in any standard local chart near the boundary, for any $\bar{\varepsilon}<\varepsilon$.

Proof. Suppose that $u_{t}$ is the solution to the nonlinear heat equation with initial data $h$. Since $\|\tau(h)\|^{2}$ lies in some $L^{p}, p>1, \tau(h)$ is bounded, and $h$ has bounded energy density, it follows [7] that $u_{t}$ exists and converges to a harmonic map $u=u_{\infty}$, as $t \rightarrow \infty$. Hartman [5] showed that $\left\|u_{t}\right\|$ is a subsolution to the usual linear heat equation. Choosing $s=\varepsilon$, in Lemma 4.3, we get an infinite number of subsolutions $\left\|u_{t}\right\|-c \psi$, any $c \geq 0$. If $c$ is large enough, then, at $t=0,\left\|u_{t}\right\|-c \psi=\|\tau(h)\|-c \psi<0$, by the decay estimate for $\tau(h)$ in Proposition 4.2. The maximum principle gives $\left\|\tau\left(u_{t}\right)\right\|<c \psi$, for all $t$. The general existence theorem of [7] states that $\left\|\tau\left(u_{t}\right)\right\|<c_{1} e^{-c_{2} t}$, for some positive constants $c_{1}$ and $c_{2}$.

Thus, for any $T$,

$$
d(h, u) \leq \int_{0}^{\infty}\left\|u_{t}\right\| d t=\int_{0}^{T}\left\|u_{t}\right\| d t+\int_{0}^{\infty}\left\|u_{t}\right\| d t .
$$

The conclusion follows by choosing $T$ of order $-\log \psi$, for points near the ideal boundary at infinity, $\partial M^{\prime}$.

Suppose that the image $\bar{M}$ is a hyperbolic space of constant negative curvature -1 . In this case, the regularity requirement of Theorem 4.4 may be significantly lowered. The analogue of Lemma 4.1 is

Lemma 4.5. Suppose $h \in C^{1, \varepsilon}\left(M^{\prime}, \bar{M}^{\prime}\right) \cap C^{2}(M, \bar{M}), 0<\varepsilon<1$, with $\bar{M}$ of constant negative curvature -1 . Such $h$ satisfies the following conditions, at the boundary:

(i) $\left(n_{1}+2 n_{2}\right)\left(h_{0}^{0}\right)^{2}-\sum_{j=0}^{n_{1}} \sum_{\gamma=1}^{\bar{n}_{1}} h_{j}^{\gamma} h_{j}^{\gamma}=0$,

(ii) $h_{0}^{0} h_{0}^{\alpha}=0, \alpha \geq 1$, 
whenever $\tau^{\alpha}(h)=O\left(y^{1+\varepsilon}\right), \alpha \geq 0$. Conversely, suppose that for the Euclidean norm, in any local coordinate chart, $\left|\nabla^{2} h\right|=O\left(y^{\varepsilon-1}\right)$. If $h_{0}^{0}>0$ and conditions (i), (ii) both hold, then $\tau^{\alpha}(h)=O\left(y^{1+\varepsilon}\right), \alpha \geq 0$.

Proof. If $\tau^{\alpha}(h)=O\left(y^{1+\varepsilon}\right)$, then we proved (i), (ii) in $\S 3$, for $h \in C^{1}\left(M^{\prime}, \bar{M}^{\prime}\right) \cap$ $C^{2}(M, \bar{M})$. Conversely, the hypothesis $\left|\nabla^{2} h\right|=O\left(y^{\varepsilon-1}\right)$, shows that the second derivative terms in (2.5a) are of order $y^{1+\varepsilon}$. If $h_{0}^{0}>0$, then (i) gives the vanishing of the first derivative terms, in formula $(2.5 \mathrm{a})$ for $\tau^{0}(h)$, up to order $y^{1+\varepsilon}$. Similarly, (ii) handles the first derivative terms for $\tau^{\alpha}(h), \alpha \geq 1$.

Following our earlier scheme, we construct an asymptotically harmonic map, given appropriate boundary data.

Proposition 4.6. Assume that $f \in C^{1, \varepsilon}\left(\partial M^{\prime}, \partial \bar{M}\right), 0<\varepsilon<1$, satisfies

$$
\sum_{j=1}^{n_{1}} \sum_{\gamma=1}^{\bar{n}_{1}} f_{j}^{\gamma} f_{j}^{\gamma}>0 .
$$

Then there exists $h \in C^{1, \varepsilon}\left(M^{\prime}, \bar{M}^{\prime}\right) \cap C^{2}(M, \bar{M})$, assuming the boundary values $f$ continuously, with $\|\tau(h)\|=O\left(y^{\varepsilon}\right)$, the Riemannian norm, of our space $\bar{M}$ with constant negative curvature.

Proof. Denote

$$
\phi=\left[\sum_{j=1}^{n_{1}} \sum_{\gamma=1}^{\bar{n}_{1}} \frac{\bar{f}_{j}^{\gamma} f_{j}^{\gamma}}{n_{1}+2 n_{2}}\right]^{1 / 2},
$$

as suggested by the hypothesis (i) of Lemma 4.5. Clearly, $\phi \in C^{0, \varepsilon}\left(\partial M^{\prime}\right)$, and we extend $\phi$ locally by convolving with the smoothing kernel, comparable to the Poisson kernel. In contrast to the proof of Proposition 4.2, we only have $f \in C^{1, \varepsilon}$. So we must also extend $f$, by convolution with a kernel comparable to the Poisson kernel, using the components of $f$ in some chart near $\partial \bar{M}^{\prime}$. We now define

$$
h(y, n)=\left(y \phi(y, n), f(y, n)-\frac{\partial f}{\partial y}(0, n) y\right) .
$$

Elementary estimates for Poisson smoothing [11], now show that $h \in$ $C^{1, \varepsilon}\left(M^{\prime}, \bar{M}^{\prime}\right)$, as in the proof of Proposition 4.2. Moreover, $h_{0}^{0}=\phi$ and $h_{0}^{\alpha}=0, \alpha \geq 1$, at the boundary $\partial M^{\prime}$. Since $h$ has boundary values $f$, $h(0, n)=(0, f(0, n))$, conditions (i), (ii) of Lemma 4.5 are valid. The Poisson smoothing guarantees that $\left|\nabla^{2} h\right|=O\left(y^{\varepsilon-1}\right)$. Thus, Lemma 4.5 yields $\|\tau(h)\|=O\left(y^{\varepsilon}\right)$. Since $\bar{M}$ has constant negative curvature, the norm is $y^{-1}$ times the locally defined Euclidean norm. This completes the local construction, on a chart near $\partial M^{\prime}$. One patches these local solutions together using a partition of unity along $\partial M^{\prime}$. The partition functions can be chosen independent of $y$, near $\partial M^{\prime}$, so that $h_{0}^{0}=\phi$ and $h_{0}^{\alpha}=0, \alpha \geq 1$, for the globally defined $h$, in any local chart. Lemma 4.5 again gives $\left\|\tau^{\alpha}(h)\right\|=O\left(y^{\ell}\right)$.

We now invoke Lemma 4.3 and apply the same argument as in the proof of Theorem 4.4, to deduce 
Theorem 4.7. Assume that $\bar{M}$ is the simply connected complete space having constant negative curvature -1 . Let $M$ be a rank one symmetric space of noncompact type.

Suppose that $f \in C^{1, \varepsilon}\left(\partial M^{\prime}, \partial \bar{M}^{\prime}\right), 0<\varepsilon<1$, satisfies $\sum_{j=1}^{n_{1}} \sum_{\gamma=1}^{\bar{n}_{1}} f_{j}^{\gamma} f_{j}^{\gamma}>$ 0 . Then there exists a harmonic map $u: M \rightarrow \bar{M}$, which assumes the boundary values $f$, continuously. If $h$ is the map of Proposition 4.6, then the Riemannian distance from $h$ to $u$ is $O\left(y^{\bar{\varepsilon}}\right)$, for any $\bar{\varepsilon}<\varepsilon$.

Remark. By applying the arguments of [9], it suffices to assume $f \in$ $C^{2}\left(\partial M^{\prime}, \partial \bar{M}^{\prime}\right)$ in the hypothesis of Theorem 4.4. Similarly, one may suppose $f \in C^{1}\left(\partial M^{\prime}, \partial \bar{M}^{\prime}\right)$ in Theorem 4.7. We omit the details since these refinements are not needed in the subsequent sections of this paper. A more careful discussion will be given elsewhere.

\section{HIGHER ORDER APPROXIMATE SOLUTIONS AND COMPATIBILITY CONDITIONS}

Let $M$ and $\bar{M}$ be rank one Riemannian symmetric spaces of noncompact type. Suppose that $f \in C^{2, \varepsilon}\left(\partial M^{\prime}, \partial \bar{M}^{\prime}\right)$ satisfies the hypothesis of Proposition 4.2. We showed that there exists $h \in C^{2, \varepsilon}\left(M^{\prime}, \bar{M}^{\prime}\right)$, assuming the boundary values $f$ continuously, whose tension field satisfies $\|\tau(h)\|=O\left(y^{\ell}\right)$. If the boundary data is smoother, $f \in C^{l+2, \varepsilon}\left(\partial M^{\prime}, \partial \bar{M}^{\prime}\right)$, then we will modify $h$ to achieve $\|\tau(h)\|=O\left(y^{l+\varepsilon}\right)$. It is already clear, from the proof of Proposition 4.2, that the $h$ constructed there lies in $C^{l+2, \varepsilon}\left(M^{\prime}, \bar{M}^{\prime}\right)$, whenever $f \in C^{l+2, \varepsilon}\left(\partial M^{\prime}, \partial \bar{M}^{\prime}\right)$. The point is to improve the decay rate of the tension field. One proceeds by an inductive argument, which is valid as long as $l \leq n_{1}+2 n_{2}$. The breakdown after a finite number of steps is expected by analogy with the studies of related problems in [4] and [8]. These higher order approximate solutions, besides being of intrinsic interest, play an important role in our subsequent development of regularity theory.

To set up the induction, assume that $h \in C^{l+2, \varepsilon}, l \geq 1,0<\varepsilon<1$, has boundary values $f \in C^{l+2, \varepsilon}$. Suppose that $h_{0}^{0}>0 ; h_{0}^{\alpha}=0,1 \leq \alpha \leq \bar{n}_{1}+\bar{n}_{2}$, $h_{00}^{\alpha}=0, \bar{n}_{1}+1 \leq \alpha \leq \bar{n}_{1}+\bar{n}_{2} ; h_{j 0}^{\gamma}=0,1 \leq j \leq n_{1}, \bar{n}_{1}+1 \leq \gamma \leq \bar{n}_{1}+\bar{n}_{2}$. If $k<l+2$, assume that $\partial^{i-1} h_{0}^{\alpha} / \partial y^{i-1}$ are determined for $i<k$ and all $\alpha$. Moreover, suppose that $\partial^{i-1} h_{0}^{\alpha} / \partial y^{i-1}$ are determined for $i \leq k, \alpha \geq \bar{n}_{1}+1$. These modifications have been made to achieve $\left|\tau^{\alpha}(h)\right|=O\left(y^{k}\right)$, all $\alpha$; and $\left|\tau^{\alpha}(h)\right|=O\left(y^{k+1}\right), \alpha \geq \bar{n}_{1}+1$. To start the induction, with $k=2$, we use the proofs of Propositions 4.2, 4.6. Let $Q_{k}$ denote a rational function of the already determine data. Note that if $\partial^{i-1} h_{0}^{\alpha} / \partial y^{i-1}$ is determined, then so are its tangential derivatives, as long as the total number of derivatives is at most $l+2$.

The inductive argument requires some detailed calculations, starting from the formulas (2.5). It is convenient to divide the presentation into three cases, depending upon the index $\alpha$ in $\tau^{\alpha}(h)$.

Case 1. $\alpha \geq \bar{n}_{1}+1$. By formula (2.5),

$$
\tau^{\alpha}(h)=g^{j j} h_{j j}^{\alpha}+\left(1-n_{1}-2 n_{2}\right) h_{0}^{\alpha} y-4 g^{j j} h_{j}^{0} h_{j}^{\alpha} \bar{y}^{-1}
$$

where $0 \leq j \leq n_{1}+n_{2}$ is summed. Thus, by the decomposition (2.2) of the 
metric,

$$
\begin{aligned}
\tau^{\alpha}(h)= & y^{2} h_{00}^{\alpha}+\sum_{j=1}^{n_{1}} y^{2} h_{j j}^{\alpha}+\sum_{j=n_{1}+1}^{n_{1}+n_{2}} y^{4} h_{j j}^{\alpha}+\left(1-n_{1}-2 n_{2}\right) h_{0}^{\alpha} y \\
& -4\left(\frac{y^{2}}{\bar{y}}\right) h_{0}^{0} h_{0}^{\alpha}-4\left(\frac{y^{2}}{\bar{y}}\right) \sum_{j=1}^{n_{1}} h_{j}^{0} h_{j}^{\alpha}-4\left(\frac{y^{4}}{\bar{y}}\right) \sum_{j=n_{1}+1}^{n_{1}+n_{2}} h_{j}^{0} h_{j}^{\alpha} .
\end{aligned}
$$

We now separate out those terms $Q_{k}$ already fixed at an earlier step of the induction

$$
\tau^{\alpha}(h)=y^{2} h_{00}^{\alpha}+\left(1-n_{1}-2 n_{2}\right) h_{0}^{\alpha} y-4\left(y^{2} / \bar{y}\right) h_{0}^{0} h_{0}^{\alpha}+Q_{k} y^{k+1}+O\left(y^{k+1+\varepsilon}\right) .
$$

Here, we used the fact that $h_{j}^{0}=0, j \geq 1 ; h_{0}^{\alpha}=0, \alpha \geq 1$, along the boundary. Using Taylor expansion of the remaining terms, we deduce, since $h_{00}^{\alpha}=0$ at the boundary,

$$
k ! \tau^{\alpha}(h)=\left(k-n_{1}-2 n_{2}-3\right) \frac{\partial^{k} h_{0}^{\alpha}}{\partial y^{k}} y^{k+1}+Q_{k} y^{k+1}+O\left(y^{k+1+\varepsilon}\right) .
$$

If $k+1<l+2$, the remainder term is $O\left(y^{k+2}\right)$. Since $l \leq n_{1}+2 n_{2}$ and $k<l+2$, we may solve uniquely for $\partial^{k} h_{0}^{\alpha} / \partial y^{k}$, in terms of $Q_{k}$, to assure that $\tau^{\alpha}(h)=O\left(y^{k+1+\varepsilon}\right)$ and $\tau^{\alpha}(h)=O\left(y^{k+2}\right)$, as long as $k+1<l+2$.

Case 2. $1 \leq \alpha \leq \bar{n}_{1}$. Again, starting from (2.5),

$$
\begin{aligned}
\tau^{\alpha}(h)= & g^{j j} h_{j j}^{\alpha}+\left(1-n_{1}-2 n_{2}\right) h_{0}^{\alpha} y-2 g^{j j} h_{j}^{0} h_{j}^{\alpha} \bar{y}^{-1} \\
& +g^{j j} \sum_{\beta=1}^{\bar{n}_{1}} \sum_{\gamma=\bar{n}_{1}+1}^{\bar{n}_{1}+\bar{n}_{2}} a_{\alpha \beta}^{\gamma-\bar{n}_{1}} h_{j}^{\beta} h_{j}^{\gamma} \bar{y}^{-2}
\end{aligned}
$$

with $j$ summed from 0 to $n_{1}+n_{2}$. Using the local expression (2.2) for the metric,

$$
\begin{aligned}
\tau^{\alpha}(h)= & y^{2} h_{00}^{\alpha}+y^{2} \sum_{j=1}^{n_{1}} h_{j j}^{\alpha}+y^{4} \sum_{j=n_{1}+1}^{n_{1}+n_{2}} h_{j j}^{\alpha}+\left(1-n_{1}-2 n_{2}\right) h_{0}^{\alpha} y \\
& -2 y^{2} h_{0}^{0} h_{0}^{\alpha} \bar{y}^{-1}-2 y^{2} \bar{y}^{-1} \sum_{j=1}^{n_{1}} h_{j}^{0} h_{j}^{\alpha}-2 y^{4} \bar{y}^{-1} \sum_{j=n_{1}+1}^{n_{1}+n_{2}} h_{j}^{0} h_{j}^{\alpha} \\
& +y^{2} \bar{y}^{-2} \sum_{\beta=1}^{\bar{n}_{1}} \sum_{\gamma=\bar{n}_{1}+1}^{\bar{n}_{1}+\bar{n}_{2}} a_{\alpha \beta}^{\gamma-\bar{n}_{1}} h_{0}^{\beta} h_{0}^{\gamma} \\
& +y^{2} \bar{y}^{-2} \sum_{j=1}^{n_{1}} \sum_{\beta=1}^{\bar{n}_{1}} \sum_{\gamma=\bar{n}_{1}+1}^{\bar{n}_{1}+\bar{n}_{2}} a_{\alpha \beta}^{\gamma-\bar{n}_{1}} h_{j}^{\beta} h_{j}^{\gamma} \\
& +y^{4} \bar{y}^{-2} \sum_{j=n_{1}+n_{2}}^{\bar{n}_{1}} \sum_{j=n_{1}+1}^{\bar{n}_{1}+\bar{n}_{2}} \sum_{\gamma=1}^{\gamma} a_{\alpha \beta}^{\gamma-\bar{n}_{1}} h_{j}^{\beta} h_{j}^{\gamma} .
\end{aligned}
$$

Identifying certain terms $Q_{k}$ already fixed at an earlier inductive step:

$$
\tau^{\alpha}(h)=y^{2} h_{00}^{\alpha}+\left(1-n_{1}-2 n_{2}\right) h_{0}^{\alpha} y-2\left(y^{2} / \bar{y}\right) h_{0}^{0} h_{0}^{\alpha}+Q_{k} y^{k}+O\left(y^{k+\varepsilon}\right) .
$$


The hypotheses $h_{0}^{\alpha}=0, \alpha \geq 1 ; h_{00}^{\gamma}=0, \gamma \geq \bar{n}_{1}+1 ; h_{j 0}^{\gamma}=0, \gamma \geq \bar{n}_{1}+1$, $1 \leq j \leq n_{1}$, were needed here, at the boundary. Taylor expanding the relevant terms gives

$$
(k-1) ! \tau^{\alpha}(h)=\left(k-n_{1}-2 n_{2}-2\right) \frac{\partial^{k-1} h_{0}^{\alpha}}{\partial y^{k-1}} y^{k}+Q_{k} y^{k}+O\left(y^{k+\varepsilon}\right) .
$$

If $k+1<l+2$, then the remainder is of order $O\left(y^{k+1}\right)$. Since $k<l+2$ and $l \leq n_{1}+2 n_{2}$, the derivative $\partial^{k-1} h_{0}^{\alpha} / \partial y^{k-1}$ is uniquely determined, in terms of the previously known data $Q_{k}$, to give $\tau^{\alpha}(h)=O\left(y^{k+\varepsilon}\right)$ and in fact the better condition $\tau^{\alpha}(h)=O\left(y^{k+1}\right)$, as long as $k+1<l+2$.

Case 3. $\alpha=0$. Returning to (2.5), we have

$$
\begin{aligned}
\tau^{0}(h)= & g^{j j} h_{j j}^{0}+\left(1-n_{1}-2 n_{2}\right) h_{0}^{0} y-g^{j j} h_{j}^{0} h_{j}^{0} \bar{y}^{-1} \\
& +g^{j j} \sum_{\gamma=1}^{\bar{n}_{1}} h_{j}^{\gamma} h_{j}^{\gamma} \bar{y}^{-1}+g^{j j} \sum_{\gamma=\bar{n}_{1}+1}^{\bar{n}_{1}+\bar{n}_{2}} h_{j}^{\gamma} h_{j}^{\gamma}\left(2 \bar{y}^{-3}\right)
\end{aligned}
$$

where one sums $j$ from 0 to $n_{1}+n_{2}$. Breaking this into pieces corresponding to the splitting (2.2) of the metric, we have

$$
\begin{aligned}
\tau^{0}(y)= & y^{2} h_{00}^{0}+\sum_{j=1}^{n_{1}} y^{2} h_{j j}^{0}+\sum_{j=n_{1}+1}^{n_{1}+n_{2}} y^{4} h_{j j}^{0}+\left(1-n_{1}-2 n_{2}\right) h_{0}^{0} y \\
& -y^{2}\left(h_{0}^{0}\right)^{2} \bar{y}^{-1}-y^{2} \sum_{j=1}^{n_{1}} h_{j}^{0} h_{j}^{0} \bar{y}^{-1}-y^{4} \sum_{j=n_{1}+1}^{n_{1}+n_{2}} h_{j}^{0} h_{j}^{0} \bar{y}^{-1} \\
& +y^{2} \sum_{\gamma=1}^{\bar{n}_{1}}\left(h_{0}^{\gamma}\right)^{2} \bar{y}^{-1}+y^{2} \sum_{j=1}^{n_{1}} \sum_{\gamma=1}^{\bar{n}_{1}} h_{j}^{\gamma} h_{j}^{\gamma} \bar{y}^{-1} \\
& +y^{4} \sum_{j=n_{1}+1}^{n_{1}+n_{2}} \sum_{\gamma=1}^{\bar{n}_{1}} h_{j}^{\gamma} h_{j}^{\gamma} \bar{y}^{-1}+2 \sum_{\gamma=\bar{n}_{1}+1}^{\bar{n}_{1}+\bar{n}_{2}} h_{0}^{\gamma} h_{0}^{\gamma} y^{2} \bar{y}^{-3} \\
& +2 y^{2} \sum_{j=1}^{n_{1}} \sum_{\gamma=\bar{n}_{1}+1}^{\bar{n}_{1}+\bar{n}_{2}} h_{j}^{\gamma} h_{j}^{\gamma} \bar{y}^{-3}+2 y^{4} \sum_{j=n_{1}+1}^{n_{1}+n_{2}} \sum_{\gamma=\bar{n}_{1}+1}^{\bar{n}_{1}+\bar{n}_{2}} h_{j}^{\gamma} h_{j}^{\gamma} \bar{y}^{-3} .
\end{aligned}
$$

Isolating appropriate terms $Q_{k}$ which are previously determined,

$$
\begin{aligned}
\tau^{0}(h)= & y^{2} h_{00}^{0}+\left(1-n_{1}-2 n_{2}\right) h_{0}^{0} y-y^{2}\left(h_{0}^{0}\right)^{2} \bar{y}^{-1} \\
& +y^{2} \bar{y}^{-1} \sum_{j=1}^{n_{1}} \sum_{\gamma=1}^{\bar{n}_{1}} h_{j}^{\gamma} h_{j}^{\gamma}+2 y^{4} \bar{y}^{-3} \sum_{j=n_{1}+1}^{n_{1}+n_{2}} \sum_{\gamma=\bar{n}_{1}+1}^{\bar{n}_{1}+\bar{n}_{2}} h_{j}^{\gamma} h_{j}^{\gamma}+Q_{k} y^{k}+O\left(y^{k+\varepsilon}\right) .
\end{aligned}
$$

Again, we used the facts $h_{0}^{\alpha}=0, \alpha \geq 1 ; h_{00}^{\gamma}=0, h_{j 0}^{\gamma}=0, \gamma \geq \bar{n}_{1}+1$, $1 \leq j \leq n_{1}$. 
Using Taylor polynomials to estimate each remaining term gives

$$
\begin{aligned}
k ! \tau^{0}(h)= & {\left[1+k\left(k-n_{1}-2 n_{2}-2\right)-\left(\frac{1}{h_{0}^{0}}\right)^{2} \sum_{j=1}^{n_{1}} \sum_{\gamma=1}^{\bar{n}_{1}} h_{j}^{\gamma} h_{j}^{\gamma}\right.} \\
& \left.-\frac{6}{\left(h_{0}^{0}\right)^{4}} \sum_{j=n_{1}+1}^{n_{1}+n_{2}} \sum_{\gamma=\bar{n}_{1}+1}^{\bar{n}_{1}+\bar{n}_{2}} h_{j}^{\gamma} h_{j}^{\gamma}\right] \frac{\partial^{k-1} h_{0}^{0}}{\partial y^{k-1}} y^{k} \\
& +Q_{k} y^{k}+O\left(y^{k+\varepsilon}\right) .
\end{aligned}
$$

If $k+1<l+2$, the remainder is $O\left(y^{k+1}\right)$. Since $l \leq n_{1}+2 n_{2}$ and $k<l+2$, there is a unique choice for $\partial^{k-1} h_{0}^{0} / \partial y^{k-1}$, which forces $\tau^{0}(h)=O\left(y^{k+\varepsilon}\right)$, in terms of previously determined data $Q_{k}$. We have $\tau^{0}(h)=O\left(y^{k+1}\right)$, if $k+1<l+2$.

These computations form the main part of the proof of

Proposition 5.1. Suppose that $f \in C^{l+2, \varepsilon}\left(\partial M^{\prime}, \partial \bar{M}^{\prime}\right), 0 \leq l \leq n_{1}+2 n_{2}, 0<$ $\varepsilon<1$ satisfies $f_{j}^{\gamma}=0,1 \leq j \leq n_{1}, \bar{n}_{1}+1 \leq \gamma \leq \bar{n}_{1}+\bar{n}_{2}$, and

$$
\sum_{j=n_{1}+1}^{n_{1}+n_{2}} \sum_{\gamma=\bar{n}_{1}+1}^{\bar{n}_{1}+\bar{n}_{2}} f_{j}^{\gamma} f_{j}^{\gamma}>0 .
$$

Then there exists $h \in C^{l+2, \varepsilon}\left(M^{\prime}, \bar{M}^{\prime}\right)$, assuming the boundary values $f$, with $\|\tau(h)\|=O\left(y^{l+\varepsilon}\right)$. Moreover, the covariant derivatives of the tension satisfy $\left\|\nabla^{j} \tau(h)\right\|=O\left(y^{l+\varepsilon}\right)$, for $j \leq l$.

Proof. If $l=0$, this reduces to Proposition 4.2. The inductive scheme just given, for $2 \leq k \leq l+1, l \geq 1$, applies in local charts near $\partial M^{\prime}$ to give $\left|\tau^{\alpha}(h)\right|=O\left(y^{l+2+\varepsilon}\right), \alpha \geq \bar{n}_{1}+1 ;\left|\tau^{\alpha}(h)\right|=O\left(y^{l+1+\varepsilon}\right), \alpha \geq 0$. Since the metric is given by (2.2), this means that $\|\tau(h)\|=O\left(y^{l+\varepsilon}\right)$, in each chart near the boundary. However, a global solution was given in Proposition 4.2, when $l=0$. At each stage of the inductive argument, in Cases $1,2,3$, one uniquely determines the Taylor series modification of $h$. This uniqueness guarantees that the local solutions agree, to sufficiently high order in $y$, fitting together to give a global solution. The estimates for $\nabla^{j} \tau(h)$ follow from successive covariant differentiation, of the Taylor polynomial of $\tau(h)$ in $y$, using the orthonormal frame field $y \partial / \partial y, y X_{i}, y^{2} Z_{j}$. Since the coefficients, on the right-hand side of (2.4), are bounded, independent of $y$; and $h_{j}^{\gamma}=0,0 \leq j \leq n_{1}, \bar{n}_{1}+1 \leq$ $\gamma \leq \bar{n}_{1}+\bar{n}_{2}$, at the boundary, $\left\|\nabla^{j} \tau(h)\right\|_{0, \varepsilon}=O\left(y^{l+\varepsilon}\right), j \leq l$.

We may now apply the nonlinear heat equation to deform our higher order approximate solution to a harmonic map. The proof of Theorem 4.4 extends easily to give

Theorem 5.2. Suppose that $f \in C^{l+2, \varepsilon}\left(\partial M^{\prime}, \partial \bar{M}^{\prime}\right), 0 \leq l<n_{1}+2 n_{2}, 0<\varepsilon<$ 1 satisfies $f_{j}^{\gamma}=0,1 \leq j \leq n_{1}, \bar{n}_{1}+1 \leq \gamma \leq \bar{n}_{1}+\bar{n}_{2}$, and $\sum_{j=n_{1}+1}^{n_{1}+n_{2}} \sum_{\gamma=\bar{n}_{1}+1}^{\bar{n}_{1}+\bar{n}_{2}} f_{j}^{\gamma} f_{j}^{\gamma}$ $>0$. Then there exists a harmonic map $u$, which assumes the boundary values $f$ continuously, so that $d(u, h)=O\left(y^{l+\bar{\varepsilon}}\right)$, any $\bar{\varepsilon}<\varepsilon$, where $h$ is the map of Proposition 5.1.

Proof. One follows the proof of Theorem 4.4, almost verbatim, using Proposition 5.1 rather than Proposition 4.2. The case $l=n_{1}+2 n_{2}$ is excluded since 
then $s=l+\varepsilon>n_{1}+2 n_{2}$, the superharmonic function $\psi$, of Lemma 4.3, only is available when $s \leq n_{1}+2 n_{2}$.

Suppose now that the range $\bar{M}$ is a hyperbolic space of constant curvature -1 . The above construction of higher order approximation can then be modified to yield more attractive results. If the boundary data $f \in C^{1, \varepsilon}\left(\partial M^{\prime}, \partial \bar{M}^{\prime}\right)$, $0<\varepsilon<1$, satisfies the hypothesis of Proposition 4.6, then we showed that there exists an extension $h \in C^{1, \varepsilon}\left(M^{\prime}, \bar{M}^{\prime}\right) \cap C^{2}(M, \bar{M})$, with $\|\tau(h)\|=O\left(y^{\varepsilon}\right)$. For smoother boundary values $f \in C^{l+1, \varepsilon}\left(\partial M^{\prime}, \partial \bar{M}^{\prime}\right)$, we plan to modify $h$ to achieve $\|\tau(h)\|=O\left(y^{l+\varepsilon}\right)$, as long as $l \leq n_{1}+2 n_{2}$. It is already clear, from the proof of Proposition 4.6, that the $h$ constructed there lies in $C^{l+1 . \varepsilon}\left(M^{\prime}, \bar{M}^{\prime}\right)$, whenever $f \in C^{l+1, \varepsilon}\left(M^{\prime}, \bar{M}^{\prime}\right)$. The point is to improve the decay rate of the tension field.

To set up the induction, assume that $h \in C^{l+1, \varepsilon}, l \geq 1,0<\varepsilon<1$, has continuously assumed boundary values $f \in C^{l+1, \varepsilon}$. Assume that $h_{0}^{0}>0$; $h_{0}^{\alpha}=0,1 \leq \alpha \leq \bar{n}_{1}+\bar{n}_{2} ;\left|\nabla_{0}^{l+2} h\right|=O\left(y^{\varepsilon-1}\right)$, where $\nabla_{0}$ denotes Euclidean derivatives in any local chart. If $k<l+1$, assume that $\partial^{i-1} h_{0}^{\alpha} / \partial y^{i-1}$ are determined for $i \leq k, \alpha \geq 0$. These modifications have been made to achieve $\left|\tau^{\alpha}(h)\right|=O\left(y^{k+1}\right)$, all $\alpha$. To start the induction, with $k=1$, we invoke the proof of Proposition 4.6. Let $Q_{k}$ denote a rational function of the already determine data.

Again, we use formulas (2.5a) and divide the discussion into the cases, depending upon the index $\alpha$ in $\tau^{\alpha}(h)$ :

Case 1. $\alpha \geq 1$. Quoting from (2.5a) gives

$$
\tau^{\alpha}(h)=g^{j j} h_{j j}^{\alpha}+\left(1-n_{1}-2 n_{2}\right) h_{0}^{\alpha} y-2 g^{j j} h_{j}^{0} h_{j}^{\alpha} \bar{y}^{-1}
$$

with $j$ summed from 0 to $n_{1}+n_{2}$. Separating this into pieces corresponding to the splitting (2.2) of the metric, gives

$$
\begin{aligned}
\tau^{\alpha}(h)= & y^{2} h_{00}^{\alpha}+y^{2} \sum_{j=1}^{n_{1}} h_{j j}^{\alpha}+y^{4} \sum_{j=n_{1}+1}^{n_{1}+n_{2}} h_{j j}^{\alpha}+\left(1-n_{1}-2 n_{2}\right) h_{0}^{\alpha} y \\
& -2 y^{2} h_{0}^{0} h_{0}^{\alpha} \bar{y}^{-1}-2 y^{2} \sum_{j=1}^{n_{1}} h_{j}^{0} h_{j}^{\alpha} \bar{y}^{-1}-2 y^{4} \sum_{j=n_{1}+1}^{n_{1}+n_{2}} h_{j}^{0} h_{j}^{\alpha} \bar{y}^{-1}
\end{aligned}
$$

We now identify the terms $Q_{k}$ already fixed:

$$
\tau^{\alpha}(h)=y^{2} h_{00}^{\alpha}+\left(1-n_{1}-2 n_{2}\right) h_{0}^{\alpha} y-2 y^{2} h_{0}^{0} h_{0}^{\alpha} \bar{y}^{-1}+Q_{k} y^{k+1}+O\left(y^{k+1+\varepsilon}\right) .
$$

Here, we used the fact that $h_{j}^{0}=0$, at the boundary, $j \geq 1$. Using the Taylor polynomial of the significant terms:

$$
k ! \tau^{\alpha}(h)=\left(k-n_{1}-2 n_{2}-1\right) \frac{\partial^{k} h_{0}^{\alpha}}{\partial y^{k}} y^{k+1}+Q_{k} y^{k+1}+O\left(y^{k+1+\varepsilon}\right) .
$$

If $k+1<l+1$, then the remainder is of order $O\left(y^{k+2}\right)$. Since $k<l+1$ and $l \leq n_{1}+2 n_{2}$, the derivative $\partial^{k} h_{0}^{\alpha} / \partial y^{k}$ is uniquely determined, in terms of the previously known data $Q_{k}$, to give $\tau^{\alpha}(h)=O\left(y^{k+1+\varepsilon}\right)$, and in fact the better condition $\tau^{\alpha}(h)=O\left(y^{k+2}\right)$, as long as $k+1<l+1$. 
Case 2. $\alpha=0$. Using (2.5a) again,

$$
\tau^{0}(h)=g^{j j} h_{j j}^{0}+\left(1-n_{1}-2 n_{2}\right) h_{0}^{0} y-g^{j j} h_{j}^{0} h_{j}^{0} \bar{y}^{-1}+g^{j j} \sum_{\gamma=1}^{\bar{n}_{1}} h_{j}^{\gamma} h_{j}^{\gamma} \bar{y}^{-1}
$$

where $0 \leq j \leq n_{1}+n_{2}$ is summed. Separating into pieces corresponding to the splitting (2.2) of the metric yields

$$
\begin{aligned}
\tau^{0}(h)= & y^{2} h_{00}^{0}+\sum_{j=1}^{n_{1}} y^{2} h_{j j}^{0}+\sum_{j=n_{1}+1}^{n_{1}+n_{2}} y^{4} h_{j j}^{0}+\left(1-n_{1}-2 n_{2}\right) h_{0}^{0} y \\
& -y^{2}\left(h_{0}^{0}\right)^{2} \bar{y}^{-1}-y^{2} \sum_{j=1}^{n_{1}} h_{j}^{0} h_{j}^{0} \bar{y}^{-1}-y^{4} \sum_{j=n_{1}+1}^{n_{1}+n_{2}} h_{j}^{0} h_{j}^{0} \bar{y}^{-1} \\
& +y^{2} \sum_{\gamma=1}^{\bar{n}_{1}} h_{0}^{\gamma} h_{0}^{\gamma} \bar{y}^{-1}+\sum_{j=1}^{n_{1}} \sum_{\gamma=1}^{\bar{n}_{1}} y^{2} h_{j}^{\gamma} h_{j}^{\gamma} \bar{y}^{-1} \\
& +y^{4} \sum_{j=n_{1}+1}^{n_{1}+n_{2}} \sum_{\gamma=1}^{\bar{n}_{1}} h_{j}^{\gamma} h_{j}^{\gamma} \bar{y}^{-1} .
\end{aligned}
$$

Identifying terms of type $Q_{k}$, which are already determined:

$$
\begin{aligned}
\tau^{0}(h)= & y^{2} h_{00}^{0}+\left(1-n_{1}-2 n_{2}\right) h_{0}^{0} y-y^{2}\left(h_{0}^{0}\right)^{2} \bar{y}^{-1} \\
& +y^{2} \bar{y}^{-1} \sum_{j=1}^{n_{1}} \sum_{\gamma=1}^{\bar{n}_{1}} h_{j}^{\gamma} h_{j}^{\gamma}+Q_{k} y^{k+1}+O\left(y^{k+1+\varepsilon}\right) .
\end{aligned}
$$

Here, we used the hypotheses that $h_{j}^{0}=0, j \geq 1$, and $h_{0}^{\alpha}=0, \alpha \geq 1$, along the boundary.

Expanding the relevant terms in Taylor series yields

$$
\begin{aligned}
(k+1) ! \tau^{0}(h) & \\
= & {\left[1+(k+1)\left(k-n_{1}-2 n_{2}-1\right)-\left(\frac{1}{h_{0}^{0}}\right)^{2} \sum_{j=1}^{n_{1}} \sum_{\gamma=1}^{\bar{n}_{1}} h_{j}^{\gamma} h_{j}^{\gamma}\right] \frac{\partial^{k} h_{0}^{0}}{\partial y^{k}} y^{k+1} } \\
& +Q_{k} y^{k+1}+O\left(y^{k+\varepsilon+1}\right) .
\end{aligned}
$$

If $k+1<l+1$, the remainder is $O\left(y^{k+2}\right)$. Since $k<l+1$ and $l \leq n_{1}+2 n_{2}$, the derivative $\partial^{k} h_{0}^{0} / \partial y^{k}$ is uniquely determined, in terms of already known data $Q_{k}$, to give $\tau^{0}(h)=O\left(y^{k+1+\varepsilon}\right)$, and actually $\tau^{0}(h)=O\left(y^{k+2}\right)$, when $k+1<l+1$.

Arguing as in the proof of Proposition 5.1, we employ these calculations to deduce an extension of Proposition 4.6.

Proposition 5.3. Assume that $f \in C^{l+1, \varepsilon}\left(\partial M^{\prime}, \partial \bar{M}^{\prime}\right), 0<\varepsilon<1$, satisfies $\sum_{j=1}^{n_{1}} \sum_{\gamma=1}^{\bar{n}_{1}} f_{j}^{\gamma} f_{j}^{y}>0$. Then there exists $h \in C^{l+1, \varepsilon}\left(M^{\prime}, \bar{M}^{\prime}\right) \cap C^{2}(M, \bar{M})$, $l \geq 0$, assuming the boundary values $f$ continuously, with $\|\tau(h)\|=O\left(y^{l+\varepsilon}\right)$, as long as $l \leq n_{1}+2 n_{2}$. Moreover, the covariant derivatives of the tension satisfy $\left\|\nabla^{j} \tau(h)\right\|_{0, \varepsilon}=O\left(y^{l+\varepsilon}\right)$, for $j \leq l$.

Applying the nonlinear heat equation, with initial data $h$, gives the analogue of Theorem 5.2. 
Theorem 5.4. Suppose that $\bar{M}$ is a hyperbolic space of constant negative curvature -1 . Assume that $f \in C^{l+1, \varepsilon}\left(\partial M^{\prime}, \partial \bar{M}^{\prime}\right), 0<\varepsilon<1,0 \leq l<n_{1}+2 n_{2}$, satisfies $\sum_{j=1}^{n_{1}} \sum_{\gamma=1}^{\bar{n}_{1}} f_{j}^{\gamma} f_{j}^{\gamma}>0$. Then there exists a harmonic map $u$, assuming the boundary values $f$ continuously, so that $d(u, h)=O\left(y^{l+\bar{\varepsilon}}\right)$, and $\bar{\varepsilon}<\varepsilon$, where $h$ is the map of Proposition 5.3.

\section{BOUNDARY REGULARITY}

Assume that $M$ and $\bar{M}$ are globally symmetric spaces of noncompact type and rank one. Given $f \in C^{l+2, \varepsilon}\left(\partial M^{\prime}, \partial \bar{M}^{\prime}\right)$, satisfying the hypotheses of Proposition 5.1, we extended $f$ to an asymptotically harmonic map $h \in$ $C^{l+2, \varepsilon}\left(M^{\prime}, \bar{M}^{\prime}\right)$, with $\left\|\nabla^{j} \tau(h)\right\|_{0, \varepsilon}=O\left(y^{l+\varepsilon}\right), j \leq l$. The nonlinear heat equation was then employed, in the proof of Theorem 5.2, to deform $h$ to a harmonic map $u$, with $d(u, h)=O\left(y^{l+\bar{\varepsilon}}\right)$, any $\bar{\varepsilon}<\varepsilon$, so that $u$ is asymptotically close to $h$, measured in the hyperbolic distance $d$. Clearly, $u$ assumes the boundary values $f$ continuously. It is natural to expect that $u$ will also inherit the boundary regularity of $h$. The key to our approach, to this issue, is the following:

Lemma 6.1. Let $h$ and $u$ be the maps constructed in Proposition 5.1 and Theorem 5.2, respectively. If $p \in M$ is Euclidean distance $y$ from the boundary, then consider the representation of $h$ and $u$, relative to Riemannian normal coordinates, on unit balls $B(p, 1)$ and $B(h(p), 1)$. In Hölder norm, relative to these normal coordinates, $\|u-h\|_{l+2, \varepsilon}=O\left(y^{l+\bar{\varepsilon}}\right)$, any $\bar{\varepsilon}<\varepsilon$.

Proof. Since the metrics on $M$ and $\bar{M}$ admit transitive groups of isometries, the Christoffel symbols, and their derivatives to any order, are bounded on $B(p, 1)$ and $B(h(p), 1)$, independent of $p$. This may be seen by composing with isometries which move $p, h(p)$ back to fixed reference points. The usual coordinates representation of the tension field gives

$$
\begin{aligned}
\Delta u^{\alpha}+\left(\Gamma_{\beta \gamma}^{\alpha} \circ u\right) \frac{\partial u^{\beta}}{\partial x_{i}} \frac{\partial u^{\gamma}}{\partial x_{j}} g^{i j} & =0, \\
\Delta h^{\alpha}+\left(\Gamma_{\beta \gamma}^{\alpha} \circ h\right) \frac{\partial h^{\beta}}{\partial x_{i}} \frac{\partial h^{\gamma}}{\partial x_{j}} g^{i j} & =\tau(h) .
\end{aligned}
$$

Here $\Delta$ is the Laplace operator of the Riemannian metric on $M$. Latin indices refer to $M$ and Greek indices refer to $\bar{M}$.

The hypotheses of Proposition 5.1, and the method of construction of $h$ and $u$, show that both of these maps have bounded energy density. By Schauder theory [3], $u$ and $h$ are each bounded in $C^{1, \varepsilon}$. Since the coefficients of the tension field equation are now $C^{\varepsilon}$ bounded, $u$ and $h$ are bounded in $C^{2, \varepsilon}$ norm. A standard iteration argument then bounds $u$ and $v$ in $C^{l+2, \varepsilon}$ norm.

We let $w=u-h$. Taking the difference of the tension field equations gives

$$
\begin{aligned}
\Delta w^{\alpha} & +\left(\Gamma_{\beta \gamma}^{\alpha} \circ u\right) \frac{\partial w^{\beta}}{\partial x_{i}} \frac{\partial u^{\gamma}}{\partial x_{j}} g^{i j}+\left(\Gamma_{\beta \gamma}^{\alpha} \circ u\right) \frac{\partial h^{\beta}}{\partial x_{i}} \frac{\partial w^{\gamma}}{\partial x_{j}} g^{i j} \\
& =\left(-\Gamma_{\beta \gamma}^{\alpha} \circ u+\Gamma_{\beta \gamma}^{\alpha} \circ h\right) \frac{\partial h^{\beta}}{\partial x_{i}} \frac{\partial h^{\gamma}}{\partial x_{j}} g^{i j}-\tau(h) .
\end{aligned}
$$


This is a linear equation for $w$ with $C^{l+1, \varepsilon}$ bounded coefficients. Since $w$ and the inhomogeneous term are of order $O\left(y^{l+\bar{\varepsilon}}\right)$, in $C^{0}$ norm, Schauder theory shows that $w$ is $O\left(y^{l+\bar{\varepsilon}}\right)$ in $C^{1, \varepsilon}$ norm. The right-hand side is now bounded, in $C^{\varepsilon}$ norm, by $O\left(y^{l+\bar{\varepsilon}}\right)$. Schauder theory shows that $w$ is $O\left(y^{l+\bar{\varepsilon}}\right)$ in $C^{2, \varepsilon}$ norm. Iteration yields the desired bound $\|u-h\|_{l+2, \varepsilon}=O\left(y^{l+\bar{\varepsilon}}\right)$. larity.

We apply this lemma to deduce our main result concerning boundary regu-

Theorem 6.2. Suppose that $f \in C^{l+2, \varepsilon}\left(\partial M^{\prime}, \partial \bar{M}^{\prime}\right), 0 \leq l<n_{1}+2 n_{2}, 0<\varepsilon<$ 1 satisfies $f_{j}^{\gamma}=0,1 \leq j \leq n_{1}, \bar{n}_{1}+1 \leq \gamma \leq \bar{n}_{1}+\bar{n}_{2}$, and $\sum_{j=n_{1}+1}^{n_{1}+n_{2}} \sum_{\gamma=\bar{n}_{1}+1}^{\bar{n}_{1}+\bar{n}_{2}} f_{j}^{\gamma} f_{j}^{\gamma}$ $>0$. Then there exists a harmonic map $u$, with boundary values $f$, and $u \in$ $C^{k+1, \bar{\varepsilon}}\left(M^{\prime}, \bar{M}^{\prime}\right)$, for $-2 \leq 2 k<l-1$, any $\bar{\varepsilon}<\varepsilon$.

Proof. Let $u$ be the harmonic map constructed in Theorem 5.2 and $h$ the asymptotically harmonic map of Proposition 5.1. In local Euclidean charts, near the boundaries of the compactifications, we have $|d u-d h|=O\left(y^{l-1+\bar{\varepsilon}}\right)$, by Lemma 6.1. The factor -1 enters because the metric (2.2) is not isotropic.

For higher derivatives, we consider the orthonormal frame field $y \partial / \partial y$, $y X_{i}, y^{2} Z_{j}$ on $M$, with its complete Riemannian metric, and the corresponding frame field on the image $\bar{M}$. Formula (2.4), for covariant derivatives in the frame field, has constant coefficients on the right-hand side. Therefore, it is comparable to the Riemannian normal coordinate frame fields used in Lemma 6.1. It follows, by induction in $k$, that $\left|V_{1} V_{2} \cdots V_{k}(d u-d h)\right|=O\left(y^{l+\bar{\varepsilon}}\right)$, where $d u-d h$ is realized as a matrix in the Riemannian orthonormal frame fields, and each $V_{i}$ belongs to our chosen orthonormal frame field.

We now convert to the Euclidean reference frame $\partial / \partial y, X_{i}, Z_{j}$. If each $W_{s} \in\left\{\partial / \partial y, X_{i}, Z_{j}\right\}$, then $\left|W_{1} W_{2} \cdots W_{k}(d u-d h)\right|=O\left(y^{l-1+\bar{\varepsilon}-2 k}\right)$, where $d u-d h$ is realized as a matrix in the Euclidean frame. The factor 2 enters, in the exponent, because of differentiations is the directions $Z_{j}$, which correspond to $y^{2} Z_{j}$ in the Riemannian orthonormal frame field. As long as, $2 k<l-1$, we see that $u$ and $h$ agree, along the boundary, up to order $k+1$.

Suppose now that the range $\bar{M}$ is a hyperbolic space of constant negative curvature -1 . In this case, we apply similar arguments, starting with Theorem 5.4, to deduce

Theorem 6.3. Assume that $\bar{M}$ is of constant negative curvature. Let $f \in$ $C^{l+1, \varepsilon}\left(\partial M^{\prime}, \partial \bar{M}^{\prime}\right), 0<\varepsilon<1,0 \leq l<n_{1}+2 n_{2}$, satisfy the condition

$$
\sum_{j=1}^{n_{1}} \sum_{\gamma=1}^{\bar{n}_{1}} f_{j}^{\gamma} f_{j}^{\gamma}>0 .
$$

Then there exists a harmonic map $u$, assuming the boundary values $f$, and moreover $u \in C^{k+1, \bar{\varepsilon}}\left(M^{\prime}, \bar{M}^{\prime}\right)$, for $-2 \leq 2 k<l-1$, for $\bar{\varepsilon}<\varepsilon$.

If both $M$ and $\bar{M}$ have constant negative curvature, the same argument gives a different proof of the following result from [8].

Theorem 6.4. Suppose that the hypotheses of Theorem 6.3 are satisfied and in addition that $M$ has constant negative curvature. Then $u \in C^{l+1, \bar{\varepsilon}}\left(M^{\prime}, \bar{M}^{\prime}\right)$, for any $\bar{\varepsilon}<\varepsilon$. 
Proof. Both the metrics on $M$ and $\bar{M}$ are conformal to the Euclidean metrics in our local charts, by comparable factors, since $h_{0}^{0}>0$. Thus $\mid d u-$ $d h \mid=O\left(y^{l+\bar{\varepsilon}}\right)$, in the Euclidean sense. For the higher derivatives we use $V_{s} \in\left\{y \partial / \partial y, y X_{i}\right\}$ and $W_{s} \in\left\{\partial / \partial y, X_{i}\right\}$. The factor 2 , from the directions $Z_{j}$, no longer appears. Thus $\left|W_{1} W_{2} \cdots W_{k}(d u-d h)\right|=O\left(y^{l+\bar{\varepsilon}-k}\right)$, allowing us to choose $k \leq l$.

\section{BIBLIOGRAPHY}

1. A. Borel, Stable real cohomology of arithmetic groups, Ann. Sci. Ecole Norm. Sup. 7 (1974), 235-272.

2. J. Eells and J. H. Sampson, Harmonic mappings of Riemannian manifolds, Amer. J. Math. 86 (1964), 109-160.

3. D. Gilbarg and N. S. Trudinger, Elliptic partial differential equations of second order, Springer-Verlag, Berlin and Heidelberg, New York, 1977.

4. R. Graham, The Dirichlet problem for the Bergman Laplacian. I, II, Comm. Partial Differential Equations 8 (1983), 433-476, 563-641.

5. P. Hartman, On homotopic harmonic mappings, Canad. J. Math. 19 (1967), 673-687.

6. S. Helgason, Differential geometry and symmetric spaces, Academic Press, San Diego, 1962.

7. $\mathrm{P} . \mathrm{Li}$ and $\mathrm{L}$. F. Tam, The heat equation and harmonic maps of complete manifolds, Invent. Math. 105 (1991), 1-46.

8. Uniqueness and regularity of proper harmonic maps, Ann. of Math. (2) 137 (1993), 167-201.

9. $\ldots$, Uniqueness and regularity of proper harmonic maps. II, Indiana J. Math. 42 (1993), 591-635.

10. R. Schoen and S. T. Yau, Compact group actions and the topology of manifolds with nonpositive curvature, Topology 18 (1979), 361-380.

11. E. M. Stein, Singular integrals and differentiability properties of functions, Princeton Univ. Press, Princeton, NJ, 1970.

Department of Mathematics, Purdue University, West Lafayette, Indiana 47907 